See discussions, stats, and author profiles for this publication at: https://www.researchgate.net/publication/271504097

\title{
Wide localized solutions of the parity-time-symmetric nonautonomous nonlinear Schrödinger equation
}

Article in Physical Review E - January 2015

DOI: 10.1103/PhysRevE.91.013205

CITATIONS

3

4 authors:

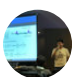

Luis Enrique Arroyo Meza

São Paulo State University

7 PUBLICATIONS 50 CITATIONS

SEE PROFILE

Marcelo Hott

São Paulo State University

57 PUBLICATIONS 581 CITATIONS

SEE PROFILE

Some of the authors of this publication are also working on these related projects:

Application of quantum mechanics to condensed matter physics View project

Nonlinear Dirac equations View project

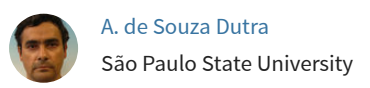
133 PUBLICATIONS 1,394 CITATIONS SEE PROFILE

(a) Pinaki Roy

Ton Duc Thang University 159 PUBLICATIONS 1,868 CITATIONS

SEE PROFILE 


\title{
Wide localized solutions of the parity-time-symmetric nonautonomous nonlinear Schrödinger equation
}

\author{
L. E. Arroyo Meza, ${ }^{1, *}$ A. de Souza Dutra, ${ }^{1, \dagger}$ M. B. Hott,,${ }^{1, \dagger}$ and P. Roy ${ }^{2, \S}$ \\ ${ }^{1}$ UNESP Universidade Estadual Paulista, Campus de Guaratinguetá, Departamento de Física e Química, \\ 12516-410 Guaratinguetá, São Paulo, Brazil \\ ${ }^{2}$ Physics and Applied Mathematics Unit, Indian Statistical Institute, Kolkata 700108, India \\ (Received 22 May 2014; revised manuscript received 30 September 2014; published 20 January 2015)
}

\begin{abstract}
By using canonical transformations we obtain localized (in space) exact solutions of the nonlinear Schrödinger equation (NLSE) with cubic and quintic space and time modulated nonlinearities and in the presence of timedependent and inhomogeneous external potentials and amplification or absorption (source or drain) coefficients. We obtain a class of wide localized exact solutions of NLSE in the presence of a number of non-Hermitian parity-time $(\mathcal{P} \mathcal{T})$-symmetric external potentials, which are constituted by a mixing of external potentials and source or drain terms. The exact solutions found here can be applied to theoretical studies of ultrashort pulse propagation in optical fibers with focusing and defocusing nonlinearities. We show that, even in the presence of gain or loss terms, stable solutions can be found and that the $\mathcal{P} \mathcal{T}$ symmetry is an important feature to guarantee the conservation of the average energy of the system.
\end{abstract}

DOI: 10.1103/PhysRevE.91.013205

PACS number(s): 05.45.Yv, 11.30.Er, 42.65.Tg, 42.65.Wi

\section{INTRODUCTION}

Nonlinear physical phenomena with losses (absorptions) and/or gains (amplifications) have been objects of extensive experimental and theoretical studies for quite a long time. Nonlinear physical phenomena can be found in many branches of physics as, for example, in semiconductor electronics [1], nonlinear optics [2], Bose-Einstein condensate (BEC) [3], and plasma physics [4], to mention a few. Usually, the dynamics of these physical phenomena can be theoretically described by a nonlinear Schrödinger equation (NLSE) with loss or gain terms. The construction of exact analytical solutions of the NLSE with loss or gain terms, including solitary waves, also called "dissipative solitons" [5], is an important and essential area of contemporary research. Exact analytical solutions of NLSE furnishes us a better understanding of those physical phenomena with loss or gain terms, especially when the theoretical model bears the main features of the real system under experimental analysis. Moreover, they help us to design parameters of control to avoid information losses carried by optical solitons and to trap BEC, while enhancing its amplitude as well as the lasting of its lifetime.

Essentially, the nonlinearity and losses and/or gains in a physical system arise from interactions between their various parts or from some inherent properties of their constituent components, as mentioned in Ref. [5]. Nowadays, due to scientific and technological advances, one has improved the capability of suitably managing the intensity of those interactions and inherent properties. In this vein, the nonlinear differential equations that model the dynamics of nonlinear physical phenomena with losses and/or gains present coefficients modulated in space-time. In this case one talks about the so-called generalized nonautonomous NLSE [6]. Modulation

\footnotetext{
*luisarroyo@feg.unesp.br

†dutra@feg.unesp.br

${ }^{\ddagger}$ marcelo.hott@pq.cnpq.br

${ }^{\S}$ rpinak@gmail.com
}

of these coefficients allows us to control the width, amplitude, and center of mass, while maintaining the overall shapes of the solutions. Theoretically, it would be important to analyze whether symmetries of the equation of motion, besides the intrinsic nonautonomy which seems to be a crucial ingredient, might also play relevant role to guarantee the shapes of solutions in dissipative systems.

The theoretical study of those systems is carried out, in general, by the analysis of the solutions (soliton solutions in particular) of a nonautonomous NLSE with cubic and quintic nonlinearities (CQNLSE) and with gain or loss term in two dimensions, given by

$$
\begin{aligned}
& i \frac{\partial \Psi}{\partial Z}+m(X, Z) \frac{\partial^{2} \Psi}{\partial X^{2}}+v(X, Z) \Psi+g_{3}(X, Z)|\Psi|^{2} \Psi \\
& \quad+g_{5}(X, Z)|\Psi|^{4} \Psi+i w(X, Z) \Psi=0
\end{aligned}
$$

where $\Psi=\Psi(X, Z)$.

From a physical point of view, the dimensionless Eq. (1) can be associated with the ultrashort pulse propagating in optical fibers, where $X$ and $Z$ stand for the transverse and longitudinal directions of propagation, respectively. $\Psi(X, Z)$ is the complex envelope of the electrical field, $m(X, Z)$ is the dispersion parameter, $v(X, Z)$ is the linear refractive index, $g_{3}(X, Z)$ and $g_{5}(X, Z)$ are the strength management of the Kerr and non-Kerr nonlinearities along the medium, respectively, and $w(X, Z)$ is the gain or loss coefficient. Likewise, Eq. (1) can govern the dynamics of BECs, where $\Psi(X, Z)$ plays the role of the macroscopic order parameter which evolves in time $Z$ and must be localized in space $X ; m(X, Z)$ is the effective mass of the condensate, $v(X, Z)$ is the coefficient associated with trapping potential, $g_{3}(X, Z)$ and $g_{5}(X, Z)$ are the strength management of the interactions between two atoms (cubic nonlinearity) and three atoms (quintic nonlinearity) [7], respectively, and $w(X, Z)$ is the gain or loss potential phenomenologically incorporated, for example, to take into account that interaction of a particle with the trapping potential is not absolutely elastic [8]. In the case of BECs one 
can also interpret the term $w(X, Z)|\Psi(X, Z)|^{2}$, in the continuity equation, as a source or drain of particles in the system.

In the past few years, a number of efficient methods were developed to obtain localized exact solutions of the generalized nonautonomous NLSE in two dimensions, such as the Hirota bilinear method [9], the similarity transformation method, which was applied for the first time to solve the nonautonomous CQNLSE in Ref. [10], and later in Refs. [11,12], and the point canonical transformations [13], which explains the origin of the ansatz involved in the similarity transformation method.

Meanwhile, recently there has been an increasing interest in studying linear and nonlinear physical systems with potentials exhibiting parity-time $(\mathcal{P} \mathcal{T})$ symmetry. This class of physical systems was introduced in the seminal paper by Bender and Boettcher [14]. The definitions of $\mathcal{P} \mathcal{T}$-symmetric potentials and their properties have been discussed in the past few years [14-17]. Physical systems exhibiting $\mathcal{P} \mathcal{T}$ symmetry have motivated investigations on several fronts in physics [18-22]. In particular, it has been suggested that in nonlinear optics there is a possibility of realizing $\mathcal{P} \mathcal{T}$-symmetric structures [23-26], which are achieved through a suitable distribution of the regions of gain or loss in the environment [23]. Specifically, in Ref. [24] the authors found a class of nonlinear self-trapped modes and examined in detail, for the first time, the interplay between nonlinearity and $\mathcal{P} \mathcal{T}$ symmetry. In Ref. [25] the authors found dissipative localized modes in both self-focusing and defocusing media with $\mathcal{P} \mathcal{T}$ symmetry. Furthermore, BECs confined in $\mathcal{P} \mathcal{T}$-symmetric potentials, both in realistic setups as well as in theoretical models, have been recently studied. In Ref. [27], the authors proposed a real quantum system in which a condensate is confined in a double-well potential with particles being injected into the condensate in one of the wells and removed from the condensate in the other potential well. Moreover, Cartarius and Wunner [20] found solutions in an arrangement of a BEC in two $\mathcal{P} \mathcal{T}$-symmetric $\delta$-function traps, without source or drain terms.

Motivated by the above discussions, we consider nonautonomous CQNLSEs with loss or gain terms $[w(X, Z)]$ that, together with the real trapping potential for BECs or with the linear and real part of the refractive index for ultrashort pulse propagation in optical fibers $[v(X, Z)]$, comprises a non-Hermitian potential in the NLSE. This nonlinear equation is useful for describing, for example, the ultrashort pulse propagation in dissipative nonlinear optical fibers $[24,25,28,29]$ or the dynamics of trapped cigar-shaped BEC by taking into account particles in a potential which is not completely elastic. Specifically, the nonautonomous CNLSE with loss or gain terms describes the dynamics of a pulse propagating in a dissipative Kerr nonlinear medium, whereas the nonautonomous CQNLSE with loss or gain terms describes the dynamics of an ultrashort pulse propagating in a dissipative non-Kerr nonlinear medium. Furthermore, the nonautonomous CQNLSE with loss or gain terms also models BECs when interactions between two atoms (cubic nonlinearity) and three atoms (quintic nonlinearity) and interactions not absolutely elastic (loss or gain term) are concurrently considered.

Here our objective is to obtain localized (in space) exact solutions of CQNLSE with loss or gain term and variable coefficients modulated in space-time. The systems we consider here possess some degree of autonomy attributable to the choices of the time-independent $\mathcal{P} \mathcal{T}$-symmetric potentials and the value of the constant nonlinearities, as explained below. Moreover, we notice, without proving it, that the $\mathcal{P} \mathcal{T}$ symmetry present in the systems we deal with is an important feature that ensures the steadiness of the solutions as well as the maintenance of their shapes during propagation. We employ point canonical transformations to map nonautonomous CQNLSE with loss or gain term, Eq. (1), onto a time-independent CQNLSE with loss or gain term whose exact solutions are known. This method was recently employed [13] to reduce a CQNLSE with space and time modulated nonlinearities onto a stationary NLSE with constant coefficients, namely,

$$
\mu \Phi=-\frac{\partial^{2} \Phi}{\partial x^{2}}+G_{3}|\Phi|^{2} \Phi+G_{5}|\Phi|^{4} \Phi,
$$

with $\Psi(X, Z)=\rho(X, Z) \exp (i \varphi(X, Z)) \Phi(x(X, Z))$ and $G_{3}$ and $G_{5}$ constants and homogeneous coefficients.

Based on the success of the point canonical transformation method applied also in the study of Schrödinger equations with time and space-dependent non-Hermitian potential [30], we resort to a slight modification of the method [31] to map Eq. (1) onto the stationary CQNLSE with a non-Hermitian $\mathcal{P} \mathcal{T}$-symmetric potential of the form

$$
\begin{gathered}
i \frac{\partial \psi}{\partial z}+\frac{\partial^{2} \psi}{\partial x^{2}}+[V(x)+i W(x)] \psi \\
+G_{3}|\psi|^{2} \psi+G_{5}|\psi|^{4} \psi=0,
\end{gathered}
$$

where $\psi(x, z)$ is related to $\Psi(X, Z)$ by a similarity transformation as shown in the next section. We remark that the very same point canonical transformations are applied to reach both Eqs. (2) and (3) and, at first view, the main modifications in the approach seems to be in the similarity transformation relating $\Psi(X, Z)$ with $\Phi(x)$ and the one relating $\Psi(X, Z)$ to $\psi(x, z)$. As a matter of fact, one can verify by comparing Sec. II of Ref. [13] with Sec. II of the present work that the system described Eq. (3) possesses some degree of autonomy concerning the choices of the $\mathcal{P} \mathcal{T}$-symmetric external potential $V(x)+i W(x)$, which constitutes part of the potential $v(X, Z)$ and part of the gain or loss term $w(X, Z)$ in the original system whose dynamics is governed by Eq. (1), while in Eq. (2) the information about the external potential $v(X, Z)$ is hidden in the behavior of $\Phi(x(X, Z))$ and in the chemical potential; that is, the external potential enjoys a lesser degree of autonomy.

The method is explained in detail in the next section, where we also discuss the particular case in which the dispersion parameter $m(X, Z)$ and the nonlinearity coefficients $g_{3}(X, Z)$ and $g_{5}(X, Z)$ depend only on $Z$. In such particular cases the form of the non-Hermitian "potential" $v(X, Z)+i w(X, Z)$ is simplified and Eq. (1) becomes more manageable. In addition we can recover most of the situations treated in the literature concerning Eq. (3) that seem to be relevant in real-world applications. In Sec. III we use the formalism described in Sec. II to obtain solutions of nonautonomous CQNLSE with some specific $\mathcal{P} \mathcal{T}$-symmetric external potentials. We work out three different examples that are generalizations 
of previously studied cases of NLSE with $\mathcal{P} \mathcal{T}$-symmetric external potentials. Such a generalization comes in two ways: the generalizations of the potentials $V(x)+i W(x)$ in Eq. (3), and the introduction of the quintic nonlinearities in problems with $\mathcal{P} \mathcal{T}$-symmetric external potentials in NLSEs. As far as we know, quintic nonlinearities - the last term in Eq. (3) - has not been considered up to now. The quintic nonlinearity accounts for three-body interaction in BECs and for corrections to the refraction index in media where ultrashort pulses propagate. By having in mind the case of $\mathrm{BECs}$, we conjecture that the injection of particles in one side of a double-well trapping potential concurrently to the removal of particles on the other well of the potentials can be implemented with a gain or loss term which, together with the trapping double-well potential, constitutes a $\mathcal{P} \mathcal{T}$-symmetric potential.

In all cases we are able to find only bright solitonlike solutions, due to the boundary conditions $\Psi(X \rightarrow \pm \infty, Z)=$ 0 imposed on the wave functions. That should be confronted with the cases when one deals with (2), for which one may find bright, dark, as well as gray solitons [13]. In Sec. IV, we analyze numerically the stability of the localized exact solutions we have obtained. We employ numerical simulations based on operator splitting Crank-Nicolson algorithm.

Finally, the Sec. V is devoted to further comments and to the conclusions.

\section{METHOD OF SOLUTION}

The specific forms of the coefficients $m(X, Z), v(X, Z), g_{3}(X, Z), g_{5}(X, Z)$, and $w(X, Z)$ in Eq. (1) are taken to be

$$
\begin{aligned}
m(X, Z) & =\frac{\zeta(Z)}{[\gamma(Z) h]^{2}}, \\
v(X, Z) & =\omega_{1}(X, Z) X^{2}+f_{1}(X, Z) X+f_{2}(X, Z)+\zeta(Z) V[F(h)]-\frac{\zeta(Z)}{h^{2}}\left[\left(\frac{h_{\xi}}{2 h}\right)^{2}-\frac{d}{d \xi}\left(\frac{h_{\xi}}{2 h}\right)\right], \\
g_{3}(X, Z) & =G_{3} \frac{\zeta(Z)}{\beta(Z)} h, \quad g_{5}(X, Z)=G_{5} \frac{\zeta(Z)}{\beta^{2}(Z)} h^{2}, \\
w(X, Z) & =f_{3}(X, Z) X+f_{4}(X, Z)+\zeta(Z) W[F(h)]
\end{aligned}
$$

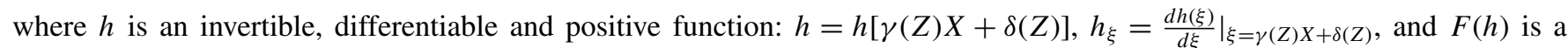
function of $h[\xi]$. The reason for choosing the nonhomogeneous coefficients and the potential in this way is clarified below.

We now perform a coordinate transformation and time rescaling [13,30,32],

$$
X=\frac{\xi}{\bar{\gamma}(z)}-\frac{\bar{\delta}(z)}{\bar{\gamma}(z)}, \quad Z-Z_{0}=\int_{0}^{z} \frac{d z^{\prime}}{\bar{\zeta}\left(z^{\prime}\right)},
$$

with $\bar{\gamma}[z(Z)]=\gamma(Z), \bar{\delta}[z(Z)]=\delta(Z)$, and $\bar{\zeta}[z(Z)]=\zeta(Z)$ and, in order to remove the first derivative of $\Psi$ with respect to $\xi$, which arises from the transformation (8), we redefine the wave function $\Psi(X, Z)$ as

$$
\Psi[X(\xi, z), Z(z)]=\sqrt{\beta(z)} e^{-i \alpha(\xi, z)} \Phi(\xi, z),
$$

where $\alpha(\xi, z)=-a(z)+\frac{1}{2} \int_{0}^{\xi} h^{2}\left(\xi^{\prime}\right)\left[\frac{\bar{\gamma}_{z}}{\bar{\gamma}}\left(\xi^{\prime}-\bar{\delta}\right)+\bar{\delta}_{z}\right] d \xi^{\prime}$, with $a(z)$ being an arbitrary function. By substituting Eqs. (4)-(9) into Eq. (1), one gets

$$
\begin{aligned}
& i \bar{\zeta} \frac{\partial \Phi}{\partial z}+\frac{\bar{\zeta}}{h^{2}} \frac{\partial^{2} \Phi}{\partial \xi^{2}}+\left\{\left[\omega_{1}+\frac{\bar{\zeta}(h)^{2}}{4} \bar{\gamma}_{z}^{2}\right]\left(\frac{\xi-\bar{\delta}}{\bar{\gamma}}\right)^{2}+\left[f_{1}+\frac{\bar{\zeta}(h)^{2}}{2} \bar{\gamma}_{z} \bar{\delta}_{z}\right]\left(\frac{\xi-\bar{\delta}}{\bar{\gamma}}\right)\right. \\
& +f_{2}+\frac{\bar{\zeta}(h)^{2}}{4} \bar{\delta}_{z}^{2}+\frac{\partial}{\partial z}\left\{-a+\frac{1}{2} \int_{0}^{\xi} h^{2}\left(\xi^{\prime}\right)\left[\frac{\bar{\gamma}_{z}}{\bar{\gamma}}\left(\xi^{\prime}-\bar{\delta}\right)+\bar{\delta}_{z}\right] d \xi^{\prime}\right\}+\bar{\zeta} V[F(h)] \\
& \left.\quad-\frac{\bar{\zeta}}{h^{2}}\left[\left(\frac{h_{\xi}}{2 h}\right)^{2}-\frac{d}{d \xi}\left(\frac{h_{\xi}}{2 h}\right)\right]\right\} \Phi+G_{3} \bar{\zeta} h|\Phi|^{2} \Phi+G_{5} \bar{\zeta} h^{2}|\Phi|^{2} \Phi \\
& +i\left\{f_{4}-\frac{h_{\xi} \bar{\zeta}}{h} \bar{\delta}_{z}+\left(f_{3}-\frac{h_{\xi}}{h} \bar{\zeta}_{z}\right)\left(\frac{\xi-\bar{\delta}}{\bar{\gamma}}\right)+\frac{\bar{\zeta}}{2} \frac{d}{d z} \ln \left(\frac{\beta}{\bar{\gamma}}\right)+\bar{\zeta} W[(h)]\right\} \Phi=0,
\end{aligned}
$$

where $f_{k}=f_{k}(\xi, z)(k=1,2,3,4)$. From the last equation one can see why the factors involving $\gamma(Z), \zeta(Z), \beta(Z)$, and $h[\gamma(Z) X+\delta(Z)]$ are present in the expressions of $v(X, Z), m(X, Z), g_{3}(X, Z)$, and $g_{5}(X, Z)$ and why we have chosen the specific dependence of $h, V$, and $W$ on $\xi=\gamma(Z) X+\delta(Z)$. Now, one may choose $\bar{\gamma}(z), \bar{\delta}(z), \bar{\zeta}(z), \bar{\beta}(z)$, and $h(\xi)$ such that

$$
\begin{aligned}
\omega_{1} & =-\frac{\bar{\zeta} h^{2}}{4} \bar{\gamma}_{z}^{2}, \quad f_{1}=-\frac{\bar{\zeta} h^{2}}{2} \bar{\gamma}_{z} \bar{\delta}_{z}, \\
f_{2} & =-\frac{\bar{\zeta} h^{2}}{4} \bar{\delta}_{z}^{2}-\bar{\zeta} \frac{\partial}{\partial z}\left\{-a+\frac{1}{2} \int_{0}^{\xi} h^{2}\left(\xi^{\prime}\right)\left[\frac{\bar{\gamma}_{z}}{\bar{\gamma}}\left(\xi^{\prime}-\bar{\delta}\right)+\bar{\delta}_{z}\right] d \xi^{\prime}\right\}, \\
f_{3} & =\frac{h_{\xi} \bar{\zeta}_{h}}{h} \bar{\gamma}_{z} \quad \text { and } \quad f_{4}=\frac{h_{\xi} \bar{\zeta}_{\bar{\delta}}}{h}-\frac{\bar{\zeta}}{2} \frac{d}{d z} \ln \left(\frac{\beta}{\bar{\gamma}}\right) .
\end{aligned}
$$


In terms of the original variables $(X, Z)$, the functions $\omega_{1}, f_{i}, i=1,2,3,4$ are given by

$$
\begin{aligned}
\omega_{1}(X, Z) & =\frac{h^{2} \gamma_{Z}^{2}}{4 \zeta(Z)}, \quad f_{1}(X, Z)=\frac{h^{2}}{2 \zeta(Z)} \gamma_{Z} \delta_{Z}, \\
f_{2}(X, Z) & =\frac{h^{2}}{4 \zeta(Z)} \delta_{Z}^{2}-\frac{\partial}{\partial Z}\left[-a+\frac{\gamma}{2 \zeta} \int_{0}^{X} h^{2}\left(\xi^{\prime}\right)\left(\gamma_{Z} X^{\prime}+\delta_{Z}\right) d X^{\prime}\right], \\
f_{3}(X, Z) & =\frac{h_{\xi}}{h} \gamma_{Z} \quad \text { and } \quad f_{4}=\frac{h_{\xi}}{h} \delta_{Z}-\frac{1}{2} \frac{d}{d Z} \ln \left(\frac{\beta}{\gamma}\right),
\end{aligned}
$$

where $\gamma_{Z}=\frac{d \gamma}{d Z}, \delta_{Z}=\frac{d \delta}{d Z}$, revealing the intrinsic connection between $\omega_{1}(X, Z), f_{k}(X, Z)(k=1,2,3,4)$ on the functions $\gamma(Z)$, $\delta(Z), \zeta(Z)$, and $\beta(Z)$ and $h[\xi=\gamma(Z) X+\delta(Z)]$. Thus, Eq. (10) takes the form

$$
i \frac{\partial \Phi}{\partial z}+\frac{1}{h^{2}} \frac{\partial^{2} \Phi}{\partial \xi^{2}}+G_{3} h|\Phi|^{2} \Phi+G_{5} h^{2}|\Phi|^{4} \Phi+i W[F(h)] \Phi+\left\{V[F(h)]-\frac{1}{h^{2}}\left[\left(\frac{h_{\xi}}{2 h[\xi]}\right)^{2}-\frac{d}{d \xi}\left(\frac{h_{\xi}}{2 h}\right)\right]\right\} \Phi=0
$$

and the wave function (9) is written as

$$
\Psi(X, Z)=\sqrt{\beta(Z)} e^{-i \alpha(X, Z)} \Phi[\xi(X, Z), z(Z)],
$$

where $\alpha(X, Z)=-a(Z)+\frac{\gamma(Z)}{2 \zeta(Z)} \int_{0}^{X} h^{2}\left(\xi^{\prime}\right)\left(\gamma_{Z} X^{\prime}+\delta_{Z}\right) d X^{\prime}$.

Since we still have a CQNLSE with nonhomogeneous nonlinearities, gain or loss coefficient, and potential, we are going to make further transformations in order to arrive at a CQNLSE with constant nonlinearities and nonhomogeneous gain or loss coefficient and potential. For that, we redefine $\xi$ as a function of another variable $x$

$$
\xi-\xi_{0}=\int_{x_{0}}^{x} \frac{d x^{\prime}}{\bar{h}\left(x^{\prime}\right)}
$$

where $\bar{h}(x)=h[\xi(x)]$ and $x-x_{0}=F(h)=\int_{\xi_{0}}^{\xi} h\left(\xi^{\prime}\right) d \xi^{\prime}$. In order to remove the first derivative of $\Phi$ with respect to $x$, which arises from the transformation (15), we redefine the wave function $\Phi$ as

$$
\Phi(\xi(x), z)=\frac{\psi(x, z)}{\sqrt{\bar{h}(x)}} .
$$

By substituting Eqs. (15) and (16) into Eq. (13), one can check that $\psi \equiv \psi(x, z)$ satisfies Eq. (3), where $V(x)=$ $V[F(h)]$ and $W(x)=W[F(h)]$. Note that the variable $z$ can be formally identified with the temporal variable and Eq. (3) can be interpreted as a CQNLSE with non-Hermitian potential $V(x)+i W(x)$. We are going to consider cases for which the potential $V(x)+i W(x)$ is invariant under the $\mathcal{P} \mathcal{T}$ transformation, that is, $x \rightarrow-x, z \rightarrow-z$, and $i \rightarrow-i$ [33].

By returning to the original space-time coordinates $(X, Z)$, the wave function can be obtained from Eqs. (16) and (14); that is,

$$
\Psi(X, Z)=\sqrt{\frac{\beta(Z)}{h[\xi(X, Z)]}} e^{-i \alpha(X, Z)} \psi(x, Z),
$$

where $\alpha(X, Z)=-a(Z)+\frac{\gamma(Z)}{2 \zeta(Z)} \int_{0}^{X} h^{2}\left(\xi^{\prime}\right)\left(\gamma_{Z} X^{\prime}+\delta_{Z}\right) d X^{\prime}$.

Thus, we have shown, by means of point canonical transformations, how the nonautonomous and nonhomogeneous CQNLSE with gain or loss term, Eq. (1), can be mapped onto a CQNLSE with constant nonlinearities and non-Hermitian potential, Eq. (3).
Particular case $h[\xi]=1$. In this case the nonautonomous and nonhomogeneous CQNLSE (1) is written as

$$
\begin{aligned}
& i \frac{\partial \Psi}{\partial Z}+m(Z) \frac{\partial^{2} \Psi}{\partial X^{2}}+v(X, Z) \Psi+g_{3}(Z)|\Psi|^{2} \Psi \\
& \quad+g_{5}(Z)|\Psi|^{4} \Psi+i w(X, Z) \Psi=0 .
\end{aligned}
$$

The coefficients are reduced to

$$
\begin{gathered}
m(Z)=\frac{\zeta(Z)}{\gamma^{2}(Z)}, \\
v(X, Z)=-\gamma \frac{d}{d Z}\left(\frac{\gamma_{Z}}{\zeta}\right) \frac{X^{2}}{4}-\gamma \frac{d}{d Z}\left(\frac{\delta_{Z}}{\zeta}\right) \frac{X}{2} \\
+\frac{d a}{d Z}+\frac{\delta_{Z}^{2}}{4 \zeta}+\zeta(Z) V(\xi), \\
g_{3}(Z)=G_{3} \frac{\zeta(Z)}{\beta(Z)}, \quad g_{5}(Z)=G_{5} \frac{\zeta(Z)}{\beta^{2}(Z)}, \\
w(X, Z)=-\frac{1}{2} \frac{d}{d Z} \ln \left(\frac{\beta}{\gamma}\right)+\zeta(Z) W(\xi),
\end{gathered}
$$

where $\xi=\gamma(Z) X+\delta(Z)$. From Eq. (15) we deduce that $\xi=$ $x$. Thus, by means of Eq. (8) and the wave function (17), which can be rewritten as

$$
\begin{aligned}
\Psi(X, Z)= & \sqrt{\beta(Z)} \psi(x, Z) \\
& \times \exp \left\{-i\left[\frac{\gamma \gamma_{Z}}{4 \zeta} X^{2}+\frac{\gamma \delta_{Z}}{2 \zeta} X-a(Z)\right]\right\},
\end{aligned}
$$

we can map the nonautonomous and nonhomogeneous CQNLSE with gain or loss term (18) onto a CQNLSE with constant nonlinearities and complex potential, Eq. (3).

In this work we are interested in localized exact solutions which obey the zero boundary conditions, i.e., $|\Psi|^{2} \rightarrow 0$ when $X \rightarrow \pm \infty$. We present three examples and find the corresponding localized (in $X$ coordinate) exact solutions for nonautonomous and nonhomogeneous CQNLSE with $\mathcal{P} \mathcal{T}$-symmetry external potentials. Specifically, in the first 
example, we work with the CNLSE which governs the optical wave propagation in a self-focusing Kerr nonlinear medium [24]. In the last two examples, we consider the CQNLSE and find localized exact solutions whose amplitudes and width can be modulated. In all examples we find only bright-soliton solutions as a consequence of the zero boundary conditions above and in the case of CQNLSE we are able to modulate the amplitude and width in such a way that a narrow bright soliton becomes a wide bright soliton.

\section{EXAMPLES}

In this section we present some examples for which localized exact solutions can be obtained and analyze their behaviors in terms of the original variables $X$ and $Z$. For this, some interesting and useful complex external potential with $\mathcal{P} \mathcal{T}$ symmetry and management coefficients are given with the suitable choices of $\gamma(Z), \delta(Z), h[\xi]$, and $\beta(Z)$.

Explicitly, we take $h[\xi]=e^{\xi^{2} / b^{2}}, \gamma(Z)=\frac{\gamma_{0}}{\sqrt{\varepsilon_{1}+\varepsilon_{2} \cos (v Z)}}$, $\zeta(Z)=\frac{\gamma^{2}}{2}, \beta(Z)=\frac{\beta_{0} \gamma_{0}}{\gamma}$, and $\delta(Z)=a(Z)=0$, with $\left|\varepsilon_{1}\right|>$ $\left|\varepsilon_{2}\right|$. We refer to Refs. [12,13] for more details on the functions $h[\xi]$ and $\gamma(Z)$. As we saw in the previous section, these functions are related to the management coefficients $m(X, Z)$, $g_{3}(X, Z)$, and $g_{5}(X, Z)$ by Eqs. (4) and (6), that is

$$
\begin{aligned}
& m(X, Z)=\frac{1}{2} e^{-\frac{2 \gamma_{0}^{2}}{b^{2}\left[\varepsilon_{1}+\varepsilon_{2} \cos (\nu Z)\right]} X^{2}}, \\
& g_{3}(X, Z)=\frac{\gamma_{0}^{2} G_{3} \exp \left\{\frac{\gamma_{0}^{2}}{b^{2}\left[\varepsilon_{1}+\varepsilon_{2} \cos (\nu Z)\right]} X^{2}\right\}}{2 \beta_{0}\left[\varepsilon_{1}+\varepsilon_{2} \cos (\nu Z)\right]^{3 / 2}}, \\
& g_{5}(X, Z)=\frac{\gamma_{0}^{2} G_{5} \exp \left\{\frac{2 \gamma_{0}^{2}}{b^{2}\left[\varepsilon_{1}+\varepsilon_{2} \cos (\nu Z)\right]} X^{2}\right\}}{2 \beta_{0}^{2}\left[\varepsilon_{1}+\varepsilon_{2} \cos (\nu Z)\right]^{2}} \text {. }
\end{aligned}
$$

It is important to remark that with this choice and from Eqs. (8) and (15), we obtain the relationship between the original variables $(X, Z)$ and the variables $(x, z)$, namely,

$$
\begin{aligned}
& x=\frac{b \sqrt{\pi}}{2} \operatorname{Erfi}\left[\frac{\gamma_{0}}{b \sqrt{\varepsilon_{1}+\varepsilon_{2} \cos (v Z)}} X\right], \\
& z=\frac{\gamma_{0}^{2}}{\nu \sqrt{\varepsilon_{1}^{2}-\varepsilon_{2}^{2}}} \arctan \left[\sqrt{\frac{\varepsilon_{1}-\varepsilon_{2}}{\varepsilon_{1}+\varepsilon_{2}}} \tan \left(\frac{\nu}{2} Z\right)\right],
\end{aligned}
$$

where Erfi is the imaginary error function [34]. Then, we notice that $(X, Z) \rightarrow(-X,-Z)$ implies into $(x, z) \rightarrow(-x,-z)$, such that we can establish the $\mathcal{P} \mathcal{T}$ symmetry in the examples we treat below.

In the particular case $h[\xi]=1$, and with the same functions $\gamma(Z), \zeta(Z)$, and $\beta(Z)$ chosen above, the relationship between the original variables $(X, Z)$ and the variables $(x, z)$ are

$$
\begin{aligned}
& x=\frac{\gamma_{0}}{\sqrt{\varepsilon_{1}+\varepsilon_{2} \cos (v Z)}} X, \\
& z=\frac{\gamma_{0}^{2}}{v \sqrt{\varepsilon_{1}^{2}-\varepsilon_{2}^{2}}} \arctan \left[\sqrt{\frac{\varepsilon_{1}-\varepsilon_{2}}{\varepsilon_{1}+\varepsilon_{2}}} \tan \left(\frac{v}{2} Z\right)\right] .
\end{aligned}
$$

Thus, when $(X, Z) \rightarrow(-X,-Z)$ one also has $(x, z) \rightarrow$ $(-x,-z)$. Moreover, from Eqs. (19) and (21) we get

$$
\begin{aligned}
& m(Z)=\frac{1}{2}, \quad g_{3}(Z)=\frac{G_{3} \gamma_{0}^{2}}{2 \beta_{0}\left[\varepsilon_{1}+\varepsilon_{2} \cos (\nu Z)\right]^{3 / 2}}, \\
& g_{5}(Z)=\frac{G_{5} \gamma_{0}^{2}}{2 \beta_{0}^{2}\left[\varepsilon_{1}+\varepsilon_{2} \cos (\nu Z)\right]^{2}},
\end{aligned}
$$

such that, from (24) and (25), one has $m(X, Z)=m(-X,-Z)$, $g_{3}(X, Z)=g_{3}(-X,-Z)$, and $g_{5}(X, Z)=g_{5}(-X,-Z)$.

\section{A. Example 1}

As a first example, we consider a model involving only cubic nonlinearity, i.e., $G_{5}=0$. For our purposes, we assume a $\mathcal{P} \mathcal{T}$-symmetric Scarf II potential [15,24,25],

$$
V(x)=V_{0} \operatorname{sech}^{2}(x), \quad W(x)=W_{0} \operatorname{sech}(x) \tanh (x),
$$

where $V_{0}$ and $W_{0}$ are the amplitudes of the real and imaginary parts of the potential, respectively. We want to point out that an analysis of the linear model associated with the potential of Eq. (26) was studied in Ref. [15]. There it was demonstrated that when $W_{0} \leqslant V_{0}+1 / 4$ the linear model presents a completely real energy spectrum. Moreover, the most interesting feature corresponding to the linear model is the existence of a critical value for the imaginary amplitude $W_{0}=V_{0}+1 / 4$. Above this critical value, an abrupt phase transition takes place, the $\mathcal{P} \mathcal{T}$ symmetry is spontaneously broken, and the real eigenvalue spectrum becomes partly or completely a complex spectrum. In the nonlinear model, such a restriction is not applied. Notwithstanding, one can check that the envelope of $\psi(x, z)$ is given by the lowest energy eigenfunction of the corresponding problem in the linear Schrödinger equation [24,25].

This first example, Eq. (3), with $V(x)$ and $W(x)$ given by Eq. (26) and $G_{3}=1$, can be linked to optical wave propagation in a self-focusing Kerr nonlinear medium with $\mathcal{P} \mathcal{T}$-symmetric potential [24] and it admits a nonlinear mode of the form

$$
\psi(x, z)=\sqrt{2-V_{0}+\left(\frac{W_{0}}{3}\right)^{2}} \operatorname{sech}(x) e^{i\left\{z+\frac{W_{0}}{3} \arctan [\sinh (x)]\right\}},
$$

corresponding to zero boundary condition at $x \rightarrow \pm \infty$.

In order to find the complex external potential and the wave function that solves Eq. (1), we substitute Eq. (26) in Eqs. (5) and (7), obtaining the refraction index $v(X, Z)$,

$$
\begin{aligned}
& v(X, Z)=-\frac{\left[4 \gamma_{0}^{4}+e^{\frac{4 \gamma_{0}^{2}}{b^{2}\left[\epsilon_{1}+\epsilon_{2} \cos (v Z)\right]} X^{2}} b^{4} \epsilon_{2}^{2} \nu^{2} \sin ^{2}(\nu Z)\right]}{8 b^{4}\left[\epsilon_{1}+\epsilon_{2} \cos (\nu Z)\right]^{2}} e^{-\frac{2 \gamma_{0}^{2}}{b^{2}\left[\epsilon_{1}+\epsilon_{2} \cos (v Z)\right]}} X^{2} X^{2}-\frac{b^{2} e^{\frac{2 \gamma_{0}^{2}}{b^{2}\left[\epsilon_{1}+\epsilon_{2} \cos (v Z)\right]}} X^{2} \epsilon_{2} \nu^{2} \cos (\nu Z)}{8 \gamma_{0}^{2}} \\
& +\frac{\gamma_{0}^{2} e^{-\frac{2 \gamma_{0}^{2}}{b^{2}\left[\epsilon_{1} \epsilon_{2} \cos (\nu)\right]} X^{2}}}{2 b^{2}\left[\epsilon_{1}+\epsilon_{2} \cos (\nu Z)\right]}+\frac{V_{0} \gamma_{0}^{2}}{2\left[\epsilon_{1}+\epsilon_{2} \cos (v Z)\right]} \operatorname{sech}^{2}\left\{\frac{\sqrt{\pi} b}{2} \operatorname{Erfi}\left[\frac{\gamma_{0}}{b \sqrt{\epsilon_{1}+\epsilon_{2} \cos (v Z)}} X\right]\right\},
\end{aligned}
$$


and the gain or loss coefficient $w(X, Z)$,

$$
\begin{aligned}
w(X, Z)= & \frac{\gamma_{0}^{2} \epsilon_{2} \nu \sin (v Z)}{b^{2}\left[\epsilon_{1}+\epsilon_{2} \cos (v Z)\right]^{2}} X^{2}+\frac{\epsilon_{2} \nu \sin (v Z)}{2\left[\epsilon_{1}+\epsilon_{2} \cos (v Z)\right]} \\
& +\frac{W_{0} \gamma_{0}^{2}}{2\left[\epsilon_{1}+\epsilon_{2} \cos (v Z)\right]} \operatorname{sech}\left(\frac{\sqrt{\pi} b}{2} \operatorname{Erfi}\left\{\frac{\gamma_{0}}{b \sqrt{\left[\epsilon_{1}+\epsilon_{2} \cos (v Z)\right]}} X\right\}\right) \tanh \left(\frac{\sqrt{\pi} b}{2} \operatorname{Erfi}\left\{\frac{\gamma_{0}}{b \sqrt{\left[\epsilon_{1}+\epsilon_{2} \cos (\nu Z)\right]}} X\right\}\right) .
\end{aligned}
$$

The later expressions satisfy the symmetry properties $v(X, Z)=v(-X,-Z)$ and $w(X, Z)=-w(-X,-Z)$; i.e., the refraction index and gain or loss coefficient are even and odd, respectively, with regard to $X$ and $Z$ reversals. Therefore, we can say that $v(X, Z)+i w(X, Z)$ works as a complex $\mathcal{P} \mathcal{T}$-symmetric potential and, due to the specific choices of $h[\xi], \gamma(Z)$, $\delta(Z), \zeta(Z)$, and $\beta(Z)$, the nonautonomous NLSE in Eq. (1) is invariant under "time" $(Z \rightarrow-Z, i \rightarrow-i)$ and space $(X \rightarrow-X)$ reversals. The wave function $\Psi(X, Z)$, which is the solution of Eq. (1), is obtained by substituting Eq. (27) into Eq. (17),

$$
\begin{aligned}
\Psi(X, Z)= & \sqrt{\beta_{0}\left[2-V_{0}+\left(\frac{W_{0}}{3}\right)^{2}\right]} \operatorname{sech}\left\{\frac{\sqrt{\pi} b}{2} \operatorname{Erfi}\left[\frac{\gamma_{0}}{b \sqrt{\epsilon_{1}+\epsilon_{2} \cos (v Z)}} X\right]\right\} \\
& \times\left[\epsilon_{1}+\epsilon_{2} \cos (\nu Z)\right]^{1 / 4} e^{-\frac{\gamma_{0}^{2}}{2 b^{2}\left[\epsilon_{1}+\epsilon_{2} \cos (\nu Z)\right]} X^{2}} e^{i \varphi(X, Z)},
\end{aligned}
$$

where

$$
\begin{aligned}
\varphi(X, Z)= & -\frac{b^{2} \epsilon_{2} \nu \sin (v Z)}{8 \gamma_{0}^{2}} \exp \left\{\frac{2 \gamma_{0}^{2} X^{2}}{b^{2}\left[\epsilon_{1}+\epsilon_{2} \cos (v Z)\right]}\right\}+\frac{W_{0}}{3} \tan ^{-1}\left(\sinh \left\{\frac{\sqrt{\pi} b}{2} \operatorname{Erfi}\left[\frac{\gamma_{0} X}{b \sqrt{\epsilon_{1}+\epsilon_{2} \cos (v Z)}}\right]\right\}\right) \\
& +\frac{\gamma_{0}^{2}}{\nu \sqrt{\varepsilon_{1}^{2}-\varepsilon_{2}^{2}}} \tan ^{-1}\left[\sqrt{\frac{\varepsilon_{1}-\varepsilon_{2}}{\varepsilon_{1}+\varepsilon_{2}}} \tan \left(\frac{\nu Z}{2}\right)\right] .
\end{aligned}
$$

From Eq. (30), we notice that $\Psi^{*}(-X,-Z)=\Psi(X, Z)$; that is, $\Psi(X, Z)$ is even under $X$ and $Z$ reversals.

A physically interesting situation arises in the particular case $h[\xi]=1$. In this case, the refractive index $v(X, Z)$ and the gain or loss coefficient $w(X, Z)$ are

$$
\begin{aligned}
& v(X, Z)=-\frac{\epsilon_{2} v^{2}\left\{4 \epsilon_{1} \cos (v Z)+\epsilon_{2}[3+\cos (2 v Z)]\right\}}{16\left[\epsilon_{1}+\epsilon_{2} \cos (v Z)\right]^{2}} X^{2}+\frac{V_{0} \gamma_{0}^{2}}{2\left[\epsilon_{1}+\epsilon_{2} \cos (v Z)\right]} \operatorname{sech}^{2}\left[\frac{\gamma_{0} X}{\sqrt{\epsilon_{1}+\epsilon_{2} \cos (v Z)}}\right], \\
& w(X, Z)=\frac{\epsilon_{2} v \sin (v Z)}{2\left[\epsilon_{1}+\epsilon_{2} \cos (v Z)\right]}+\frac{W_{0} \gamma_{0}^{2}}{2\left[\epsilon_{1}+\epsilon_{2} \cos (v Z)\right]} \operatorname{sech}\left[\frac{\gamma_{0} X}{\sqrt{\epsilon_{1}+\epsilon_{2} \cos (v Z)}}\right] \tanh \left[\frac{\gamma_{0} X}{\sqrt{\epsilon_{1}+\epsilon_{2} \cos (v Z)}}\right] .
\end{aligned}
$$

Even in this particular case, the refraction index and gain or loss coefficient are even and odd, respectively, with regard to $X$ and $Z$ reversals. Thus, the potential $v(X, Z)+i w(X, Z)$ is $\mathcal{P} \mathcal{T}$ symmetric.

Moreover, Eq. (18) reduces to the form

$$
i \frac{\partial \Psi}{\partial Z}+\frac{1}{2} \frac{\partial^{2} \Psi}{\partial X^{2}}+g_{3}(Z)|\Psi|^{2} \Psi+v(X, Z) \Psi+i w(X, Z) \Psi=0,
$$

where $g_{3}(Z), v(X, Z)$, and $w(X, Z)$ satisfy Eqs. (25) and (31). Equation (32) can be associated with the optical wave propagation in the self-focusing Kerr nonlinear medium with modulated refractive index along both the transverse and the longitudinal axes [35]. Kartashov and Torner discovered that, under proper conditions, longitudinally periodic lattices can exhibit amplification of spatial soliton but they did not take into account radiative losses that occur when a high-frequency wing of the soliton spatial spectrum is transmitted through the potential barrier and leaves the guiding channel [35]. By means of this example, we consider a refractive index modulated along both the transverse and longitudinal axes, similar to that considered in [35]. Besides, we take into account the losses and gains of the system and explore the effects of Kerr nonlinearity, refraction index and gain or loss coefficient on the optical wave propagation.

Figure 1 presents the plot of the refractive index $v(X, Z)$ and gain or loss coefficient $w(X, Z)$. From Fig. 1, one can see that $v(X, Z)$ forms a regular distribution of potential barriers along the longitudinal axis $Z$ [Fig. 1(a)], and each potential barrier is correlated with a sudden change of $w(X, Z)$ in the transverse direction $X$ [Fig. 1(b)]. Moreover, the strength of the non-Kerr nonlinearity, $g_{3}(Z)$, is maximum in every place where the potential barrier appears. The changes of $w(X, Z)$ are interpreted as a power outflow in one side of the fiber while a power inflow is provided by an external source of energy on the other side of the fiber, which can be appreciated by means of the continuity 


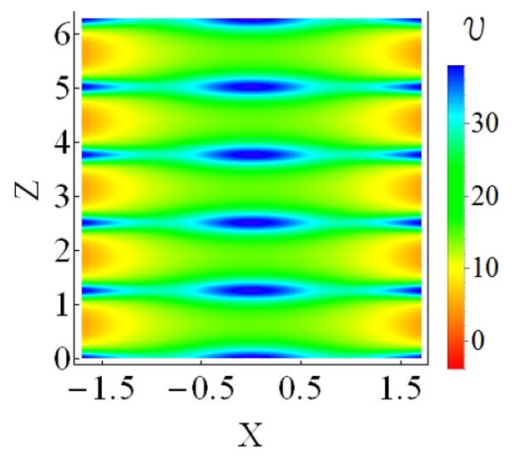

(a)

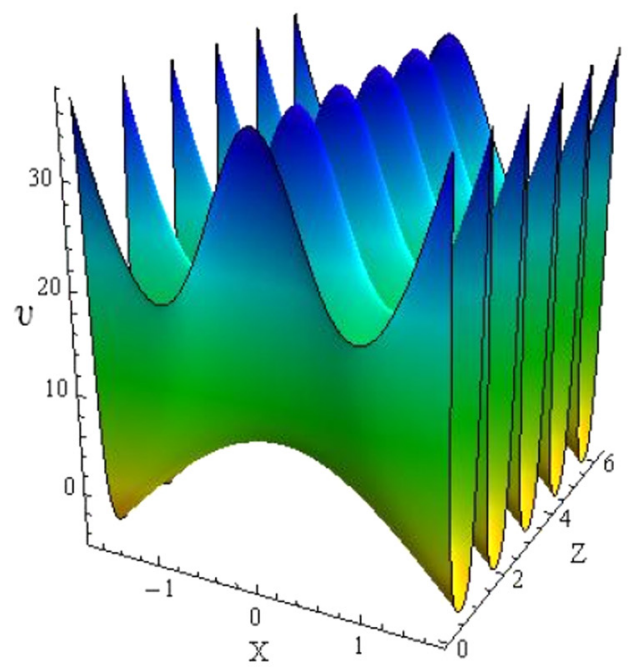

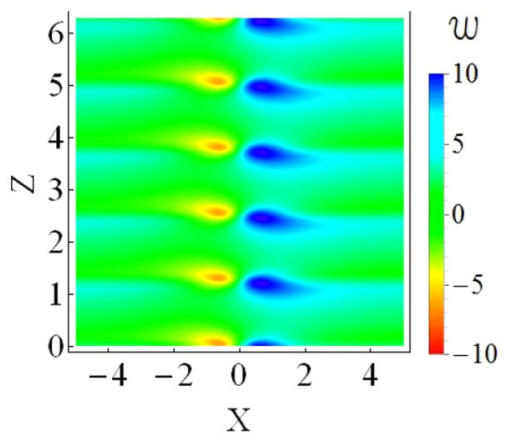

(b)

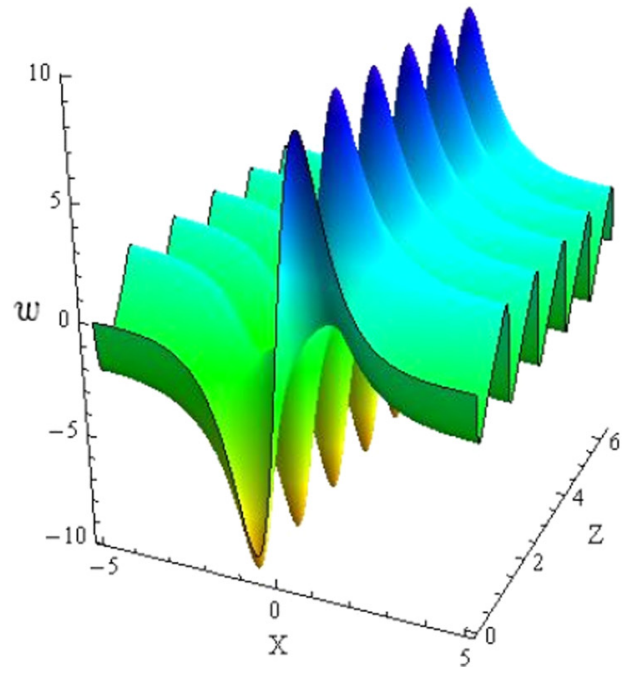

FIG. 1. (Color online) Periodic evolution of (a) the refraction index $v(X, Z)$ and (b) the gain or loss coefficient $w(X, Z)$ [Eq. (31)], with $v=5, \epsilon_{1}=6, \epsilon_{2}=-4, V_{0}=37, W_{0}=18$, and $\gamma_{0}=2$.

equation (Poynting's theorem) $\partial_{Z}|\Psi|^{2}+\partial_{X}\left[i / 2\left(\Psi \partial_{X} \Psi^{*}-\right.\right.$ $\left.\left.\Psi^{*} \partial_{X} \Psi\right)\right]=-2 w(X, Z)|\Psi|^{2}$, with $\Psi=\Psi(X, Z)$ given below.

By using Eqs. (23) and (27), we obtain the wave function $\Psi(X, Z)$, which is the solution of Eq. (32),

$$
\begin{aligned}
\Psi(X, Z)= & \sqrt{\beta_{0}\left[2-V_{0}+\left(\frac{W_{0}}{3}\right)^{2}\right]} \\
& \times \operatorname{sech}\left[\frac{\gamma_{0}}{\sqrt{\epsilon_{1}+\epsilon_{2} \cos (v Z)}} X\right] \\
& \times\left[\epsilon_{1}+\epsilon_{2} \cos (\nu Z)\right]^{1 / 4} e^{i \phi(X, Z)},
\end{aligned}
$$

where

$$
\begin{aligned}
\phi(X, Z)= & \frac{W_{0}}{3} \varphi(X, Z) \\
& +\frac{\gamma_{0}^{2}}{\nu \sqrt{\varepsilon_{1}^{2}-\varepsilon_{2}^{2}}} \arctan \left[\sqrt{\frac{\varepsilon_{1}-\varepsilon_{2}}{\varepsilon_{1}+\varepsilon_{2}}} \tan \left(\frac{v}{2} Z\right)\right] \\
& -\frac{\epsilon_{2} \nu \sin (\nu Z)}{4\left[\epsilon_{1}+\epsilon_{2} \cos (\nu Z)\right]} X^{2}
\end{aligned}
$$

with $\varphi(X, Z)=\arctan \left[\sinh \gamma_{0} X / \sqrt{\epsilon_{1}+\epsilon_{2} \cos (\nu Z)}\right]$. We notice that again $\Psi^{*}(-X,-Z)=\Psi(X, Z)$.
The evolution and behavior of the intensity of the localized exact solution $|\Psi(X, Z)|^{2}$ is shown in Fig. 2. From that figure, one can see that the intensity of the localized exact solution forms a dip due to the effects produced on it by the potential barrier and the maximum strength of $g_{3}(Z)$. The sudden change of $w(X, Z)$ does not affect $|\Psi(X, Z)|^{2}$, it affects only the phase of $\Psi(X, Z)$. The role played by the external source or drain of energy can be appreciated through the source or drain term on the right-hand side of the continuity equation (Poynting's theorem), as discussed above. We recall that the $\mathcal{P} \mathcal{T}$ symmetry imposes that $w(X, Z)$ must be odd under $X$ and $Z$ reversals and this leads to the conservation of the average energy in one period $v^{-1}$, as one can check explicitly by integrating in $X$ and by taking the average of the continuity equation. Thus, one can say that the $\mathcal{P} \mathcal{T}$ symmetry must be present in order to have stable solutions of those kind of systems.

A simpler example happens when only the potential and the gain or loss term are space-time dependent and the nonlinearity is homogeneous and time independent. By considering this case and by taking $h[\xi]=1, \gamma(Z)=\frac{\gamma_{0}}{\sqrt{\varepsilon_{1}+\varepsilon_{2} \cos [v Z]}}, \zeta(Z)=$ $\beta(Z)=\frac{\gamma^{2}}{2}$, and $\delta(Z)=a(Z)=G_{5}=0$, with $\left|\varepsilon_{1}\right|>\left|\varepsilon_{2}\right|$, Eq. (1) reduces to the form

$$
i \frac{\partial \Psi}{\partial Z}+\frac{1}{2} \frac{\partial^{2} \Psi}{\partial X^{2}}+G_{3}|\Psi|^{2} \Psi+v(X, Z) \Psi+i w(X, Z) \Psi=0
$$


(a)

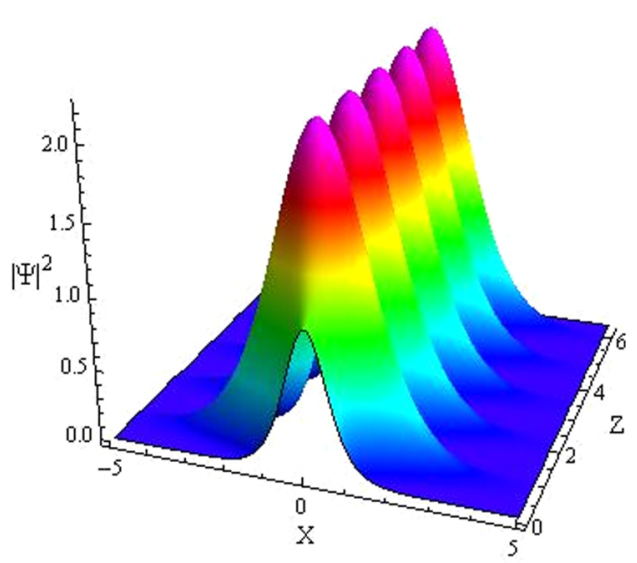

(b)

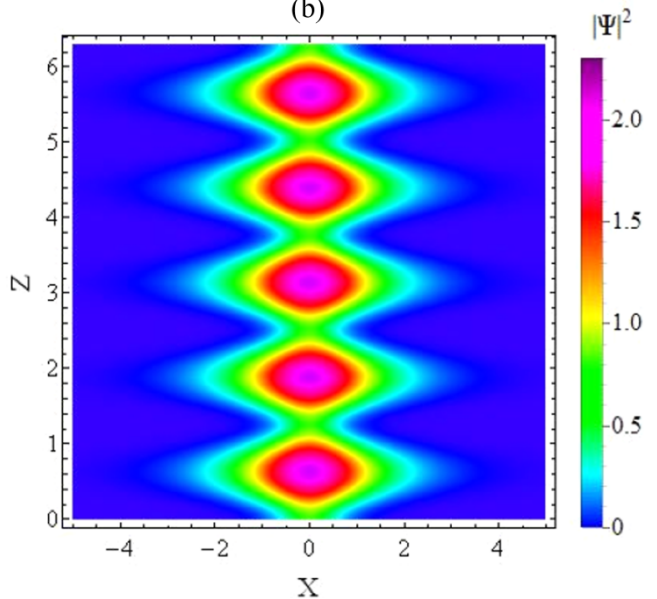

FIG. 2. (Color online) (a) $|\Psi(X, Z)|^{2}$ like a breathing bright soliton. (b) Contour plot of "breathing bright soliton," Eq. (33), with $v=5$, $\epsilon_{1}=6, \epsilon_{2}=-4, V_{0}=37, W_{0}=18, \beta_{0}=1 / \sqrt{2}$, and $\gamma_{0}=2$.

where $v(X, Z)$ and $w(X, Z)$ are the same given by Eq. (31). The solution to the latter differential equation is

$$
\begin{aligned}
\Psi(X, Z)= & \sqrt{\frac{\gamma_{0}}{2}\left[2-V_{0}+\left(\frac{W_{0}}{3}\right)^{2}\right]} \\
& \times \operatorname{sech}\left[\frac{\gamma_{0}}{\epsilon_{1}+\epsilon_{2} \cos (v Z)} X\right] \\
& \times\left[\epsilon_{1}+\epsilon_{2} \cos (\nu Z)\right]^{-1 / 2} e^{i \phi(X, Z)} .
\end{aligned}
$$

\section{B. Example 2}

In this example we present a generalization of the model considered in the previous example. Here we take into account both cubic and quintic nonlinearities. To achieve our goal, we assume a generalized $\mathcal{P} \mathcal{T}$-symmetric Scarf II potential,

$$
V(x)=\frac{2 V_{0} \lambda}{\mu+\lambda \cosh (2 x)}, \quad W(x)=\frac{(2 \lambda)^{3 / 2} W_{0} \sinh (2 x)}{2[\mu+\lambda \cosh (2 x)]^{3 / 2}},
$$

where $\mu \geqslant \lambda>0$ are parameters of the model. An interesting feature of the $\mathcal{P} \mathcal{T}$-symmetric potential in Eq. (34) is its continuous deformation as $\lambda$ varies. From Figs. 3(a) and 3(b), one can observe that the amplitudes of the real and imaginary parts decrease while spacing between the regions of loss and gain of the imaginary part increases as $\lambda$ approaches zero.

Thus, Eq. (3) with $V(x)$ and $W(x)$ given in Eq. (34) models optical wave propagation in a non-Kerr nonlinear medium with a $\mathcal{P} \mathcal{T}$-symmetric potential, and it admits, with $G_{5}=$ $-\frac{3 G_{3}^{2}(\mu+\lambda)(\mu-\lambda)}{4 \lambda^{2}\left(2 \mu / \lambda-V_{0}+W_{0}^{2} / 9\right)^{2}}$, a localized exact solution of the form

$$
\begin{aligned}
\psi(x, z)= & \sqrt{\frac{2 \lambda}{G_{3}}\left[2 \frac{\mu}{\lambda}-V_{0}+\left(\frac{W_{0}}{3}\right)^{2}\right]} \\
& \times \frac{\exp \left\{i\left[z+\frac{W_{0}}{3} \sqrt{\frac{2 \lambda}{\lambda+\mu}} F(\varphi \mid m)\right]\right\}}{\sqrt{\mu+\lambda \cosh (2 x)}},
\end{aligned}
$$

where $F(\varphi \mid m)$ is the incomplete elliptic integral of the first kind [34], with amplitude $\varphi=\arctan [\sinh (x)]$ and elliptic parameter $m=(\mu-\lambda) /(\mu+\lambda)$.

A relevant aspect of the behavior of the intensity of the localized exact solution is that its width as well as its amplitude increase when $\lambda$ approaches zero, going from a bright soliton to a wide bright soliton [13], as it is shown in Figs. 3(c) and 3(d). Otherwise, if the value of $\lambda$ increases, then the strength of the non-Kerr nonlinearity $G_{5}$ decreases to its minimum value $\frac{-3 G_{3}^{2}}{4\left[4-\left(W_{0}^{2} / 9-V_{0}\right)^{2}\right]}$ when $\lambda=\frac{\left(V_{0}-W_{0}^{2} / 9\right) \mu}{2}$; beyond that point $G_{5}$ increases to zero. The amplitude of (a)

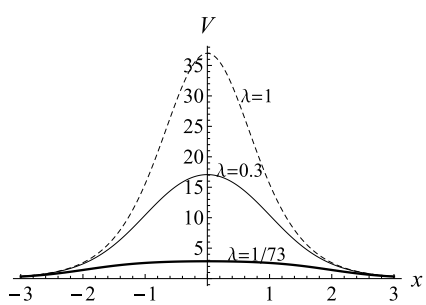

(b)

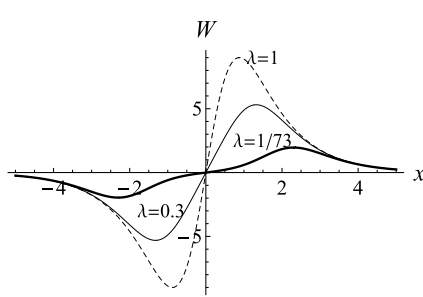

(c)

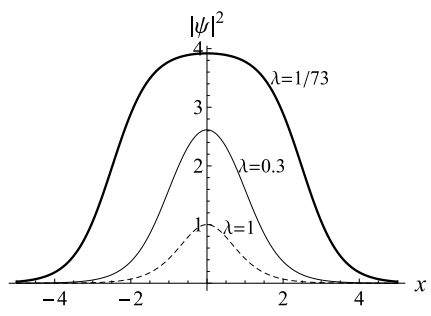

(d)

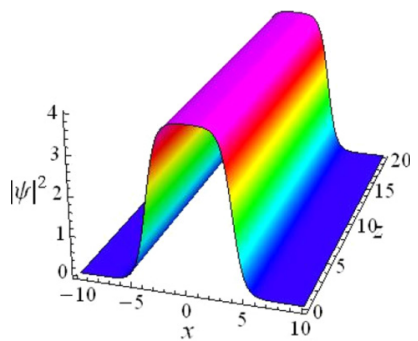

FIG. 3. (Color online) Continuous deformation of (a) $V(x)$ real part of the $\mathcal{P} \mathcal{T}$-symmetric potential [Eq. (34)]. (b) $W(x)$ imaginary part of the $\mathcal{P} \mathcal{T}$-symmetric potential [Eq. (34)]. (c) Profile of $|\psi(x)|^{2}$ [Eq. (35)] for different values of $\lambda$. (d) $|\psi(x)|^{2}$ similar to a wide bright soliton, for $\lambda=1 / 73$. With $V_{0}=37, W_{0}=18, \mu=1$. 


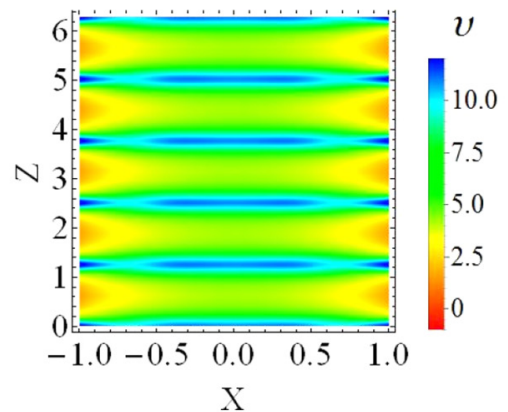

(a)

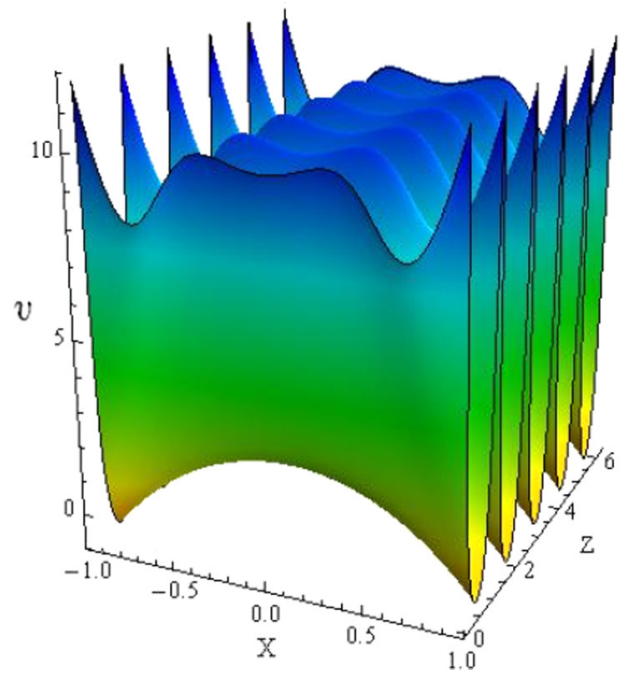

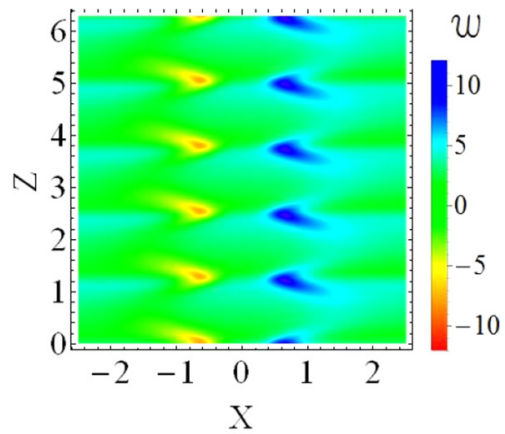

(b)

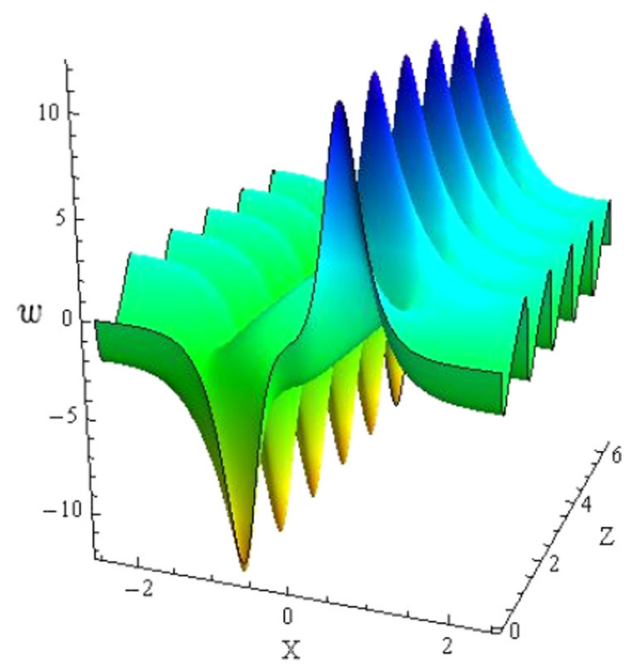

FIG. 4. (Color online) (a) Deformed refraction index $v(X, Z)$ [Eq. (36)]. (b) Continuous separation of regions of loss and gain, $w(X, Z)$ [Eq. (37)], with $v=5, \gamma_{0}=2, \epsilon_{1}=0.6, \epsilon_{2}=-0.4, V_{0}=37, W_{0}=18, \mu=1$, and $\lambda=1 / 73$.

the transverse power-flow density $S=\frac{i}{2}\left(\psi \partial_{x} \psi^{*}-\psi^{*} \partial_{x} \psi\right)=$ $\frac{W_{0}\left(2 \mu / \lambda-V_{0}+W_{0}^{2} / 9\right)}{3 G_{3}}\left[\frac{2 \lambda}{\mu+\lambda \cosh (2 x)}\right]^{3 / 2}$ associated with the above localized exact solution increases when $\lambda$ decreases.

It is noteworthy that, when $\lambda=\mu$, the non-Kerr nonlinearity vanishes $\left(G_{5}=0\right)$ and the $\mathcal{P} \mathcal{T}$-symmetric potential, Eq. (34), the localized exact solution, Eq. (35), and the transverse power-flow density $S$ are reduced precisely to those equations presented in Refs. [24,25] (first example). It is in this sense that we say the second example is a generalization of the first one. Thus, when $G_{3}=1$ (self-focusing case) or
$G_{3}=-1$ (self-defocusing case) our model is a generalization of the models given in Refs. [24,25] and Ref. [29], respectively.

We now study the evolution Eq. (18) using the generalized $\mathcal{P} \mathcal{T}$-symmetric Scarf II potential. In this case, Eq. (18) might describe the optical wave propagation in a non-Kerr nonlinear medium with refractive index and gain or loss term modulation along both transverse and longitudinal axes. From Eqs. (20), (22), and (34), we find that the refraction index $v(X, Z)$ and the gain or loss coefficient $w(X, Z)$ are

$$
\begin{gathered}
v(X, Z)=-\frac{\epsilon_{2} v^{2}\left\{4 \epsilon_{1} \cos (v Z)+\epsilon_{2}[3+\cos (2 v Z)]\right\}}{16\left[\epsilon_{1}+\epsilon_{2} \cos (v Z)\right]^{2}} X^{2}+\frac{V_{0} \gamma_{0}^{2}}{\left[\epsilon_{1}+\epsilon_{2} \cos (v Z)\right]} \frac{\lambda}{\mu+\lambda \cosh \left[\frac{2 \gamma_{0} X}{\sqrt{\epsilon_{1}+\epsilon_{2} \cos (v Z)}}\right]}, \\
w(X, Z)=\frac{\epsilon_{2} v \sin (v Z)}{2\left[\epsilon_{1}+\epsilon_{2} \cos (v Z)\right]}+\frac{W_{0} \gamma_{0}^{2}}{4\left[\epsilon_{1}+\epsilon_{2} \cos (v Z)\right]} \sinh \left[\frac{2 \gamma_{0} X}{\sqrt{\epsilon_{1}+\epsilon_{2} \cos (v Z)}}\right] \frac{(2 \lambda)^{3 / 2}}{\left\{\mu+\lambda \cosh \left[\frac{2 \gamma_{0} X}{\sqrt{\epsilon_{1}+\epsilon_{2} \cos (\nu Z)}}\right]\right\}^{3 / 2}} .
\end{gathered}
$$

From the last expressions, one can check that $v(X, Z)=$ $v(-X,-Z)$ and $w(X, Z)=-w(-X,-Z)$. Then, the complex potential $v(X, Z)+i w(X, Z)$ is $\mathcal{P} \mathcal{T}$ symmetric. In this case, the refractive index and gain or loss term have a development such as that shown in Fig. 1, but with a peculiar behavior; that is, when $\lambda$ is approaching zero the width and height of the potential barriers formed by $v(X, Z)$ are continuously deformed, which leads to regions of gain and loss that are continuously separated. This peculiar behavior is shown in Fig. 4. Moreover, the maximum strength of the Kerr and minimum 
(a)

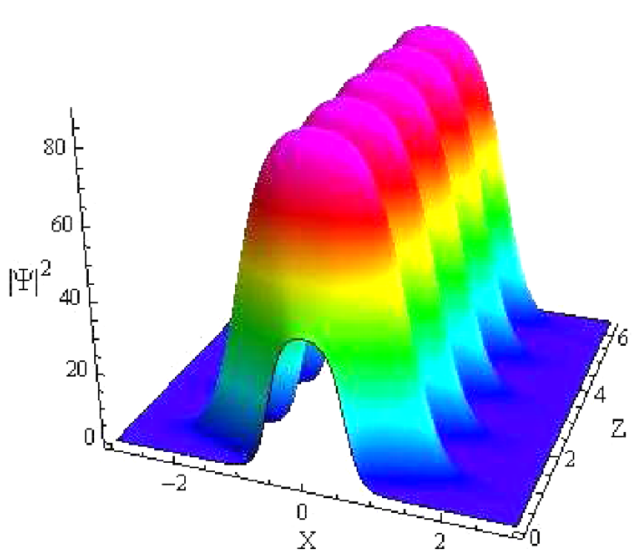

(b)

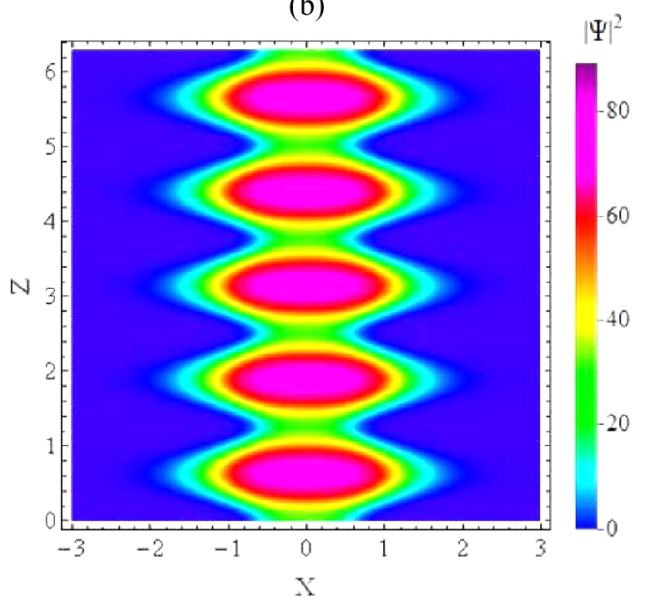

FIG. 5. (Color online) (a) Intensity, $|\Psi|^{2}$, similar to a breathing wide bright soliton. (b) Contour plot of the period evolution the "breathing wide bright soliton" (38), with $v=5, \epsilon_{1}=0.6, \epsilon_{2}=-0.4, \gamma_{0}=2, \beta_{0}=10 \sqrt{5}, V_{0}=37, W_{0}=18, \mu=1$, and $\lambda=1 / 73$.

strength of the non-Kerr nonlinearities, $g_{3}(Z)$ and $g_{5}(Z)$, are in phase with the potential barriers. Now we examine the repercussions of Kerr and non-Kerr nonlinearities and of the deformed $v(X, Z)$ and $w(X, Z)$ on the optical wave propagation.

The wave function $\Psi(X, Z)$, which is the solution of Eq. (18), is obtained from Eq. (23), using Eq. (35), and is given by

$$
\begin{aligned}
\Psi(X, Z)= & \sqrt{\frac{2 \beta_{0} \lambda}{G_{3}}\left[2 \frac{\mu}{\lambda}-V_{0}+\left(\frac{W_{0}}{3}\right)^{2}\right]} e^{i \phi(X, Z)} \\
& \times \frac{\left[\epsilon_{1}+\epsilon_{2} \cos (\nu Z)\right]^{1 / 4}}{\sqrt{\mu+\lambda \cosh \left[\frac{2 \gamma_{0} X}{\sqrt{\epsilon_{1}+\epsilon_{2} \cos (\nu Z)}}\right]}}
\end{aligned}
$$

where

$$
\begin{aligned}
\phi(X, Z)= & -\frac{\epsilon_{2} \nu \sin (\nu Z)}{4\left[\epsilon_{1}+\epsilon_{2} \cos (\nu Z)\right]} X^{2} \\
& +\frac{W_{0}}{3} \sqrt{\frac{2 \lambda}{\lambda+\mu}} F\left[\varphi(X, Z) \mid \frac{\mu-\lambda}{\mu+\lambda}\right] \\
& +\frac{\gamma_{0}^{2}}{v \sqrt{\varepsilon_{1}^{2}-\varepsilon_{2}^{2}}} \arctan \left[\sqrt{\frac{\varepsilon_{1}-\varepsilon_{2}}{\varepsilon_{1}+\varepsilon_{2}}} \tan \left(\frac{\nu Z}{2}\right)\right],
\end{aligned}
$$

with $\varphi(X, Z)=\arctan \left\{\sinh \left[\gamma_{0} X / \sqrt{\epsilon_{1}+\epsilon_{2} \cos (v Z)}\right]\right\}$. From Eq. (38) we can deduce that $\Psi^{*}(-X,-Z)=\Psi(X, Z)$.

The intensity of the beam is similar to that shown in Fig. 2. However, in this case we can also modulate the width and amplitude of the intensity by varying the parameter $\lambda$. That is, when $\lambda$ approaches zero, the width and amplitude increases due to the decreasing of the potential barriers and due to the separation of the regions of loss and gain and those effects produced by the maximum and minimum strength of the $g_{3}(Z)$ and $g_{5}(Z)$, respectively. Thus, when $\lambda$ is near zero, the intensity of the beam is similar to a wide breathing bright soliton [13], as shown in Fig. 5.

It is worth noting that, when $\lambda=\mu\left(G_{5}=0\right)$, Eqs. (39) and (38) are reduced exactly to Eqs. (31) and (33), as expected.

We also notice that the above example may be applied to the case of BEC. In that case, the equation $\partial_{Z}|\Psi|^{2}+$
$\partial_{X}\left[i / 2\left(\Psi \partial_{X} \Psi^{*}-\Psi^{*} \partial_{X} \Psi\right)\right]=-2 w(X, Z)|\Psi|^{2}$ is interpreted as governing the continuity of the number of particles in the system. Again, the odd parity of $w(X, Z)$ under $X$ and $Z$ reversals implies the conservation of the average number of particles. As a matter of fact, the term on the right-hand side of the continuity equation may be interpreted as source or drain of particles, where the supplying and draining of particles occur simultaneously and in opposite sides of the potential wells, very similar to the effect described in Ref. [27].

It is noteworthy that for the case when the nonlinear coefficients are constants, i.e., $g_{3}(Z)=G_{3}$ and $g_{5}(Z)=G_{5}$, the system also presents analytical solutions. For example, if we consider $h[\xi]=1, \gamma(Z)=\sqrt{2}, \zeta(Z)=\beta(Z)=1$, and $a(Z)=-\int \frac{\delta_{Z}^{2}}{4} d Z$, Eq. (1) takes the form

$$
\begin{aligned}
& i \frac{\partial \Psi}{\partial Z}+\frac{1}{2} \frac{\partial^{2} \Psi}{\partial X^{2}}+G_{3}|\Psi|^{2} \Psi+G_{5}|\Psi|^{4} \Psi \\
& \quad+v(X, Z) \Psi+i w(X, Z) \Psi=0
\end{aligned}
$$

where $v(X, Z)=-\frac{\delta_{Z Z}}{\sqrt{2}} X+V_{0} \operatorname{sech}^{2}[X+\delta(Z)] \operatorname{and} w(X, Z)=$ $(2 \lambda)^{3 / 2} W_{0} \frac{\sinh \{2[X+\delta(Z)]\}}{(\mu+\lambda \cosh \{2[X+\delta(Z)]\})^{3 / 2}}$, and the analytic solution of the differential equation which describes the dynamics of the system is

$$
\begin{aligned}
|\Psi(X, Z)|= & \sqrt{\frac{2 \lambda}{G_{3}}\left(\frac{2 \mu}{\lambda}-V_{0}+\frac{W_{0}^{2}}{9}\right)} \\
& \times(\mu+\lambda \cosh \{2[X+\delta(Z)]\})^{-1 / 2}
\end{aligned}
$$

The evolution of the intensity of the analytical solution in this case has a snakelike behavior, which was first studied in Ref. [31].

On the other hand, when we take $h[\xi]=1, \gamma(Z)=$ $\frac{\gamma_{0}}{\sqrt{\varepsilon_{1}+\varepsilon_{2} \cos (v Z)}}, \zeta(Z)=\beta(Z)=1$, and $\delta(Z)=a(Z)=0$, with $\left|\varepsilon_{1}\right|>\left|\varepsilon_{2}\right|$, Eq. (1) reduces to the expression

$$
\begin{aligned}
& i \frac{\partial \Psi}{\partial Z}+m(Z) \frac{\partial^{2} \Psi}{\partial X^{2}}+G_{3}|\Psi|^{2} \Psi+G_{5}|\Psi|^{4} \Psi \\
& \quad+v(X, Z) \Psi+i w(X, Z) \Psi=0,
\end{aligned}
$$


(a)

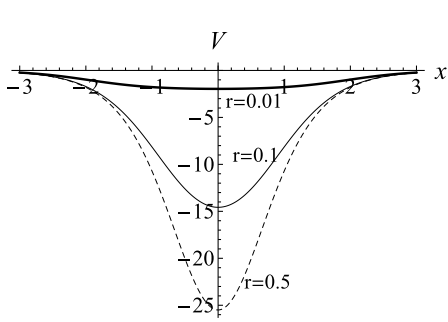

(b)

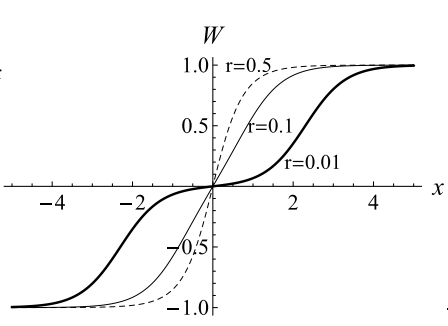

(c)

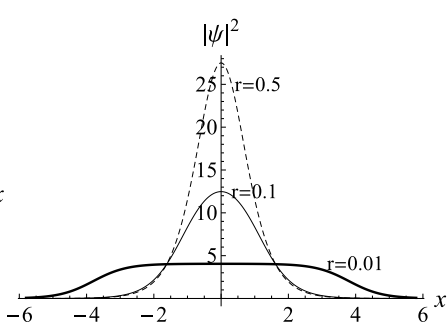

(d)

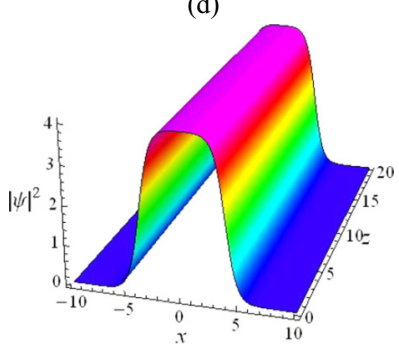

FIG. 6. (Color online) (a) Profile of the real part of the complex potential [Eq. (39)] . (b) Profile of the imaginary part of the complex potential [Eq. (39)] . (c) Transverse section of the intensity for different values of $r$. (d) Intensity of the exact solution similar to wide bright soliton [Eq. (40)] for $r=0.01$. With $V_{0}=25.5, W_{0}=0.5, s=0.5$.

where $m(Z)=\left[\varepsilon_{1}+\varepsilon_{2} \cos (\nu Z)\right] / \gamma_{0}^{2}$,

$$
\begin{aligned}
v(X, Z)= & \frac{\nu^{2} \gamma_{0}^{2} \varepsilon_{2}\left[\left(\varepsilon_{2}-4 \varepsilon_{1}\right) \cos (\nu Z)-5 \varepsilon_{2}\right]}{32\left[\varepsilon_{1}+\varepsilon_{2} \cos (\nu Z)\right]^{3}} X^{2} \\
& +V_{0} \operatorname{sech}^{2}\left[\frac{\gamma_{0} X}{\sqrt{\varepsilon_{1}+\varepsilon_{2} \cos (v Z)}}\right] \\
w(X, Z)= & \frac{\varepsilon_{2} \nu \sin (\nu Z)}{4\left[\varepsilon_{1}+\varepsilon_{2} \cos (\nu Z)\right]} \\
& +W_{0} \sinh \left[\frac{2 \gamma_{0} X}{\sqrt{\varepsilon_{1}+\varepsilon_{2} \cos (v Z)}}\right] \\
& \times \frac{(2 \lambda)^{3 / 2}}{\left\{\mu+\lambda \cosh \left[\frac{2 \gamma_{0} X}{\sqrt{\epsilon_{1}+\epsilon_{2} \cos (\nu Z)}}\right]\right\}^{3 / 2}} .
\end{aligned}
$$

The analytic solution for the latter differential equation is

$$
\begin{aligned}
|\Psi(X, Z)|= & \sqrt{\frac{2 \lambda}{G_{3}}\left(\frac{2 \mu}{\lambda}-V_{0}+\frac{W_{0}^{2}}{9}\right)} \\
& \times\left\{\mu+\lambda \cosh \left[\frac{2 \gamma_{0} X}{\sqrt{\varepsilon_{1}+\varepsilon_{2} \cos (v Z)}}\right]\right\}^{-1 / 2} .
\end{aligned}
$$

\section{Example 3}

In this last example we present a generalization of the model proposed by Midya and Roychoudhury [36], and then we analyze the evolution of $\Psi(X, Z)$ governed by Eq. (18). For our purpose, we consider a generalized $\mathcal{P} \mathcal{T}$-symmetric Rosen-Morse II potential,

$$
V(x)=-\frac{2 V_{0} r}{s+r \cosh (2 x)}, \quad W(x)=\frac{2 W_{0} r \sinh (2 x)}{s+r \cosh (2 x)},
$$

where $s \geqslant r>0$ are parameters of the generalized model. It is important to mention that the linear problem for the $\mathcal{P} \mathcal{T}$ symmetric Rosen-Morse II potential was studied by Lévai and Magyari [37]. There the authors demonstrated that the energy spectrum of the linear problem is always real and the energy eigenvalues can take positive or negative values, depending on a suitable choice of the values of the amplitudes of the real and imaginary parts of this potential. This feature might be due to the imaginary part of the potential, which in contrast to the real part is asymptotically nonvanishing. For this reason, there is no breaking of symmetry and consequently there is no phase transition in such systems.
The generalized $\mathcal{P} \mathcal{T}$-symmetric Rosen-Morse II potential is continuously deformed when $r$ approaches to zero. Figures 6(a) and 6(b) present the profiles of both real and imaginary parts of the potential $V(x)+i W(x)$ for different values of $r$.

In this case, Eq. (3) with $V(x)$ and $W(x)$ given by Eq. (39) and with $G_{5}=-\frac{3 G_{3}^{2}(s-r)(s+r)}{4\left(V_{0} r+2 s\right)^{2}}$ would describe the optical wave propagation in a non-Kerr medium with nonlinear $\mathcal{P} \mathcal{T}$ symmetric potential and it admits a localized exact solution of the form

$$
\psi(x, z)=\sqrt{\frac{2\left(V_{0} r+2 s\right)}{G_{3}}} \frac{e^{i\left[\left(1-W_{0}^{2}\right) z+W_{0} x\right]}}{\sqrt{s+r \cosh (2 x)}} .
$$

From Eq. (40), one can observe that $|\psi(x, z)|$ does not depend on $W_{0}$. Furthermore, the width and the amplitude of the intensity of the localized exact solution increases and decreases, respectively, at the same time that $r$ approaches zero, so the intensity goes from a bright solitonlike to a wide bright solitonlike, as presented in Figs. 6(c) and 6(d). On the other hand, the strength of the non-Kerr nonlinearity $G_{5}$ decreases when $r$ approaches zero and reaches its minimum value, $-\frac{3 G_{3}^{2}}{16}$, when $r=0$. Furthermore, the energy associated to the localized exact solution, $\varepsilon=\int_{-\infty}^{\infty}|\psi(x, z)|^{2} d x=$ $\frac{4\left(2 s+V_{0} r\right) \operatorname{arccoth}[\sqrt{(s+r) /(s-r)}]}{G_{3} \sqrt{(s+r) /(s-r)}}$, increases when $r$ is near zero.

Note that when $r$ equals $s$, the strength of the non-Kerr nonlinearity vanishes $\left(G_{5}=0\right)$, the $\mathcal{P} \mathcal{T}$ symmetric complex potential Eqs. (39), the localized exact solution Eq. (40), and the energy $\varepsilon$ are reduced exactly to those presented in Ref. [36]. In view of this, we say that the our model is a generalization of the model studied in aforementioned reference.

Now we study the evolution of the solution of Eq. (1) in the presence of the $\mathcal{P} \mathcal{T}$-symmetric Rosen-Morse II potential, Eq. (39), and $\beta(Z)=\frac{\beta_{0}}{\gamma_{0}} \gamma(Z)$. In this case, from Eqs. (20) and (22), we get the refraction index $v(X, Z)$ and the gain or loss coefficient $w(X, Z)$ given by

$$
\begin{aligned}
v(X, Z)= & -\frac{\epsilon_{2} v^{2}\left\{4 \epsilon_{1} \cos (v Z)+\epsilon_{2}[3+\cos (2 \nu Z)]\right\}}{16\left[\epsilon_{1}+\epsilon_{2} \cos (v Z)\right]^{2}} X^{2} \\
& -\frac{\gamma_{0}^{2}}{\epsilon_{1}+\epsilon_{2} \cos (v Z)} \frac{V_{0} r}{s+r \cosh \left[\frac{2 \gamma_{0} X}{\epsilon_{1}+\epsilon_{2} \cos (v Z)}\right]}, \\
w(X, Z)= & \frac{\gamma_{0}^{2} W_{0} r}{\epsilon_{1}+\epsilon_{2} \cos (v Z)} \frac{\sinh \left[\frac{2 \gamma_{0} X}{\epsilon_{1}+\epsilon_{2} \cos (v Z)}\right]}{s+r \cosh \left[\frac{2 \gamma_{0} X}{\epsilon_{1}+\epsilon_{2} \cos (v Z)}\right]} .
\end{aligned}
$$




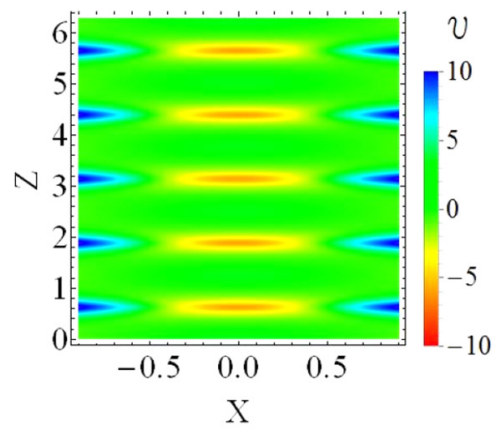

(a)

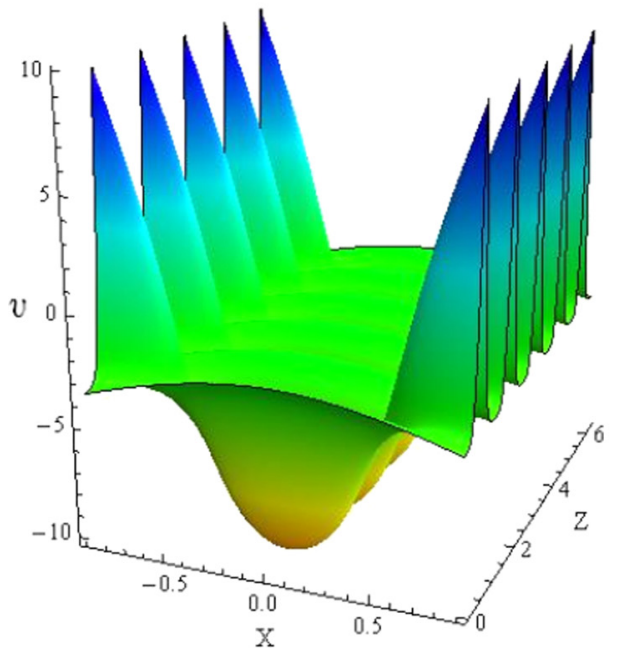

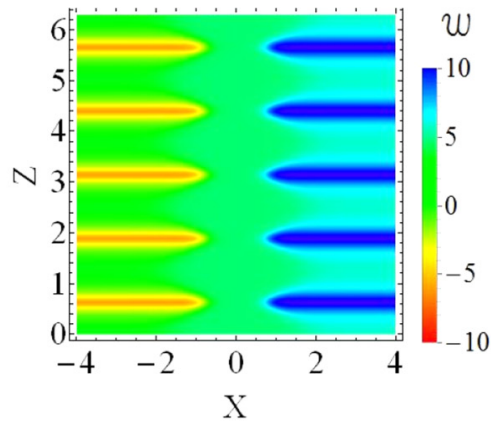

(b)

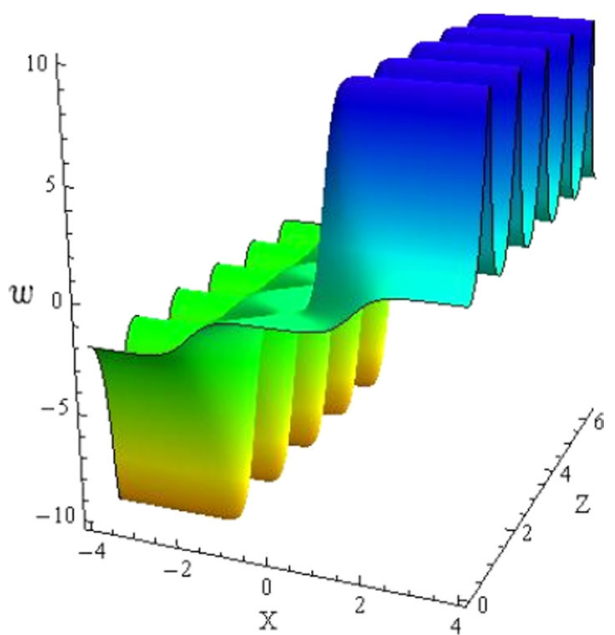

FIG. 7. (Color online) (a) Deformed refraction index. (b) Continuous separation of regions of loss and gain [Eq. (39)], with $v=5$, $\gamma_{0}=2$, $\epsilon_{1}=0.6, \epsilon_{2}=0.4, V_{0}=25.5, W_{0}=0.5, s=0.5$, and $r=0.01$.

From these last expressions it is easy to check that $v(X, Z)=$ $v(-X,-Z)$ and $w(X, Z)=-w(-X,-Z)$. In this case the complex potential $v(X, Z)+i w(X, Z)$ is $\mathcal{P} \mathcal{T}$ symmetric, too. In this context, the refraction index forms a regular distribution of potential wells along the longitudinal axis $Z$ [Fig. 7(a)], and each potential well is associated with a change of the gain or loss coefficient in the transverse axis $X$ [Fig. 7(b)]. Furthermore, the width and depth of each potential well formed by $v(X, Z)$ is continuously deformed, whereas the regions of gain and loss in $w(X, Z)$ are continuously kept apart from each other when $r$ approaches zero, as shown in Fig. 7. Also, the strength of the Kerr nonlinearity $g_{3}(Z)$ is maximum wherever the potential wells appear and non-Kerr nonlinearity $g_{5}(Z)$ is constant.

The localized exact solution $\Psi(X, Z)$, which is the solution of Eq. (1), is obtained from Eq. (23) by using Eq. (40),

$$
\begin{aligned}
\Psi(X, Z)= & \sqrt{\frac{2\left(V_{0} r+2 s\right) \beta_{0}}{G_{3}}} e^{i \varphi(X, Z)} \\
& \times \frac{\left[\epsilon_{1}+\epsilon_{2} \cos (\nu Z)\right]^{-1 / 4}}{\sqrt{s+r \cosh \left[\frac{2 \gamma_{0} X}{\epsilon_{1}+\epsilon_{2} \cos (\nu Z)}\right]}},
\end{aligned}
$$

where

$$
\begin{aligned}
\varphi(X, Z)= & -\frac{\epsilon_{2} \nu \sin (\nu Z)}{4\left[\epsilon_{1}+\epsilon_{2} \cos (\nu Z)\right]} X^{2}+\frac{W_{0} \gamma_{0}}{\sqrt{\epsilon_{1}+\epsilon_{2} \cos (\nu Z)}} X \\
& +\frac{\gamma_{0}^{2}\left(1-W_{0}^{2}\right)}{\nu \sqrt{\varepsilon_{1}^{2}-\varepsilon_{2}^{2}}} \tan ^{-1}\left[\sqrt{\frac{\varepsilon_{1}-\varepsilon_{2}}{\varepsilon_{1}+\varepsilon_{2}}} \tan \left(\frac{\nu Z}{2}\right)\right] .
\end{aligned}
$$

From Eq. (42) we see that $\Psi^{*}(-X,-Z)=\Psi(X, Z)$. The amplitude of the localized exact solution is shown in Fig. 8. From Fig. 8, one can see that the amplitude of the localized solution is maximum at the bottom of the potential well $(X=0)$, which coincides with the maximum strength of the Kerr nonlinearity and the sudden change of $w(X, Z)$. Moreover, the intensity of the wave takes the form of a wide breathing bright soliton when $r$ is close to zero (Fig. 8).

We want to draw attention to the fact that the localized exact solutions that we have presented in examples 2 and 3 cannot be recovered from any of the $\mathcal{P} \mathcal{T}$-symmetric potentials studied in Ref. [38].

\section{STABILITY ANALYSIS}

So far, we have presented new localized exact solutions of the nonautonomous CQNLSE with gain or loss term Eq. (1). 
(a)

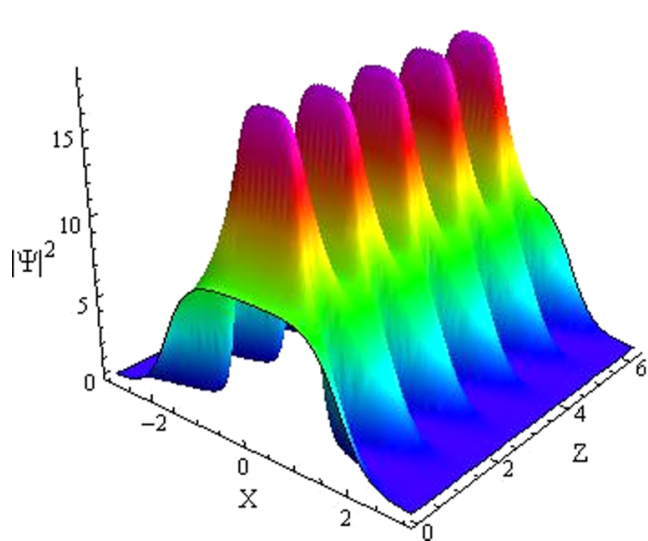

(b)

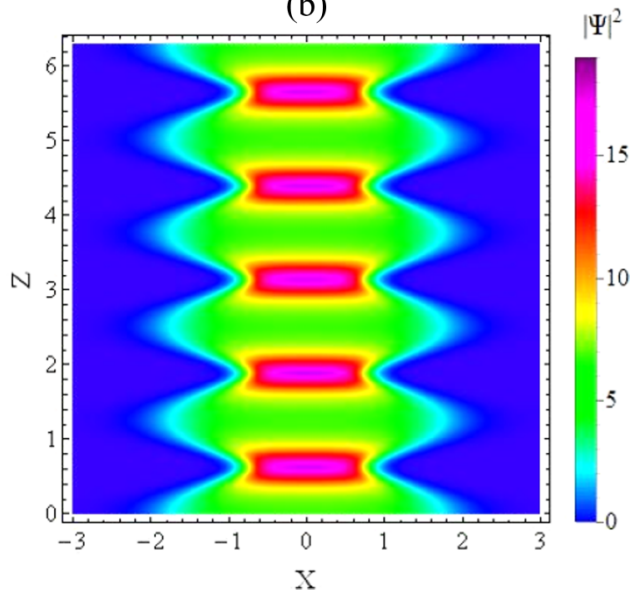

FIG. 8. (Color online) (a) $|\Psi(X, Z)|^{2}$, like "breathing wide bright soliton." (b) Contour plot of the periodic evolution of $|\Psi(X, Z)|^{2}[$ Eq. $(42)]$, with $v=5, \gamma_{0}=2, \epsilon_{1}=0.6, \epsilon_{2}=0.4, \beta_{0}=2, V_{0}=25.5, W_{0}=0.5, s=0.5$, and $r=0.01$.

It is worth mentioning that in real-world applications the stable solutions are the more important ones for the dynamics of physical phenomena.

In this section, we analyze by means of a numerical method and by resorting to Lyapunov criteria, the stability of the localized exact solutions obtained in the previous section, Eqs. (33), (38), and (42). For it, we have employed numerical simulations based on operator splitting Crank-Nicolson algorithm, by working with finite-difference methods. This approach has already been successfully employed in Ref. [39] to analyze the stability of two-component vector solitons. We refer to Refs. [40,41] for more details on the description of the algorithm by using the Mathematica software platform.

Here we use the steps $\Delta X=0.02$ and $\Delta Z=0.01$, for the transverse and longitudinal directions of propagation, respectively, to obtain the numerical results over the longitudinal direction of propagation in the range $[0,400]$. Moreover, we add a white noise in the initial pulse $\Psi(X, 0)$, in such a way that the perturbed pulse reads

$$
\Psi_{\text {pert }}(X, 0)=\Psi(X, 0)[1+0.01 R(X)]
$$

where $R(X)$ is a random number between 0 and 1 .

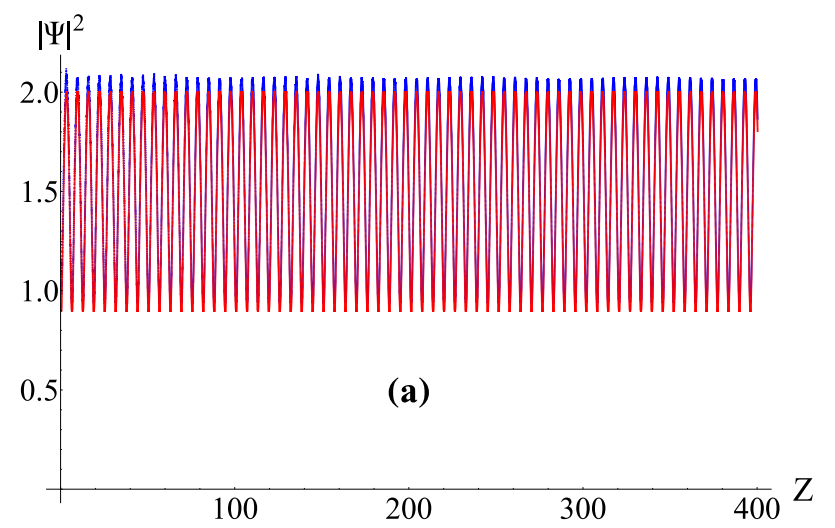

The comparison between numerical and analytical results are shown in Fig. 9, where the solutions are shown only for $X=0$. One can note that the maximum amplitudes of the perturbed numerical solutions as functions of $Z$ do not coincide with the corresponding analytical ones. In fact, according to Lyapunov criteria [42,43], when the perturbed solution evolves around the analytical one the system is said to be stable. This can be applied to both solutions (33) and (38). In the later case, the stability is realized only for $\lambda \leqslant \mu$, since that the solution becomes more susceptible to the initial perturbation, that is, it tends to be unstable as $\lambda$ approaches zero (when the quintic nonlinearity coefficient becomes predominant).

Moreover, the localized exact solution (42) was found to be unstable under the considered initial perturbation according to Lyapunov criteria, since the perturbed solution evolves away from the exact one. We have not presented the numerical findings regarding the solution (42) and, once it is unstable, it should be understood in this study only as an example of the application of the method.

\section{CONCLUSION}

In general, a complete description of the propagation of ultrashort pulses in optical fibers takes into account higher-order

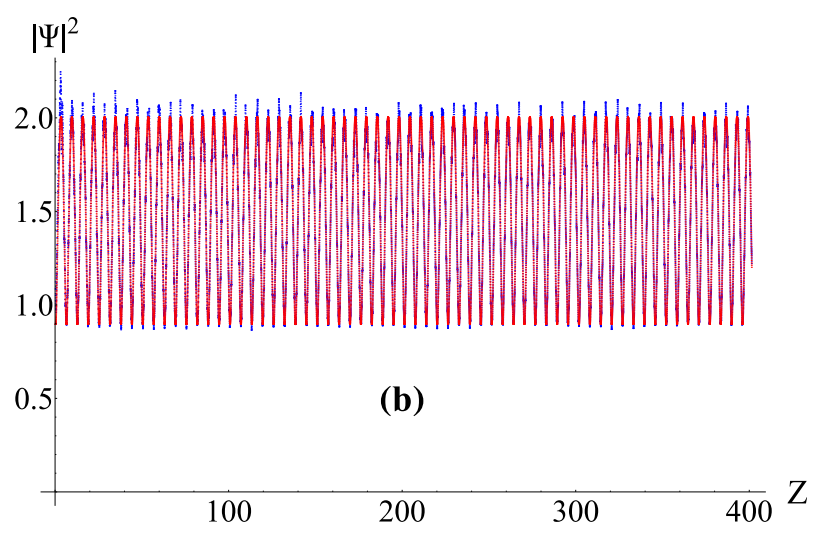

FIG. 9. (Color online) Analysis of stability. (a) Maximum amplitude of the solution Eq. (33); analytical (red) and perturbed (blue). (b) Maximum amplitude of the solution Eq. (38); analytical (red) and perturbed (blue) for $\lambda=\frac{\mu}{2}\left(V_{0}-W_{0}^{2} / 9\right)$. 
nonlinearities, which arise from the expansion of the refractive index $n$ in powers of ultrashort pulse intensity $I$, namely, $n=n_{0}+n_{2} I+n_{4} I^{2}+\cdots$, where $n_{0}$ is the linear refractive index coefficient, $n_{2}=3 \chi^{(3)} / 8 n_{0}$ and $n_{4}=5 \chi^{(5)} / 32 n_{0}$ are the cubic and the quintic nonlinearity coefficients, respectively; $\chi^{(i)}$ the $i$ th-order susceptibility. If the expansion is truncated at second-order terms with respect to $I$, the corresponding differential equation governing the nonlinear evolution of the ultrashort pulse in the optical fiber is the CQNLSE, where $n_{0}$ splits into a real and an imaginary part, which are sometimes designated as a potential and a gain or loss (amplification or absorption) term, respectively. The refraction index may depend on both the longitudinal and transverse directions of propagation and, when those coefficients are specifically designed, by managing them in order to guarantee the propagation without dissipation of the optical wave as a nondissipative soliton, one has the so-called nonautonomous CQNLSE.

In this paper we have applied point canonical transformation, together with similarity transformation, to map nonautonomous CQNLSE onto an autonomous one with a complex potential. Explicitly, we map Eq. (1) onto Eq. (3). The potential, $V(x)$, and loss or gain term, $W(x)$, in Eq. (3), comprise a non-Hermitian potential $V(x)+i W(x)$. We have dealt with $\mathcal{P} \mathcal{T}$-symmetric non-Hermitian potentials, that is, $V(-x)=V(x)$ and $i W(-x)=i W(x)$, which render Eq. (3) invariant under $x$ and $z$ reversals. By considering specific configurations of the $\mathcal{P} \mathcal{T}$-symmetric non-Hermitian potentials in the autonomous NLSE, we have been able to reproduce results previously obtained in the literature and we have also generalized them. From the wave functions that are solutions of Eq. (3), we have obtained the solutions of the nonautonomous CQNLSE. Most of the cases previously treated in the literature concerning Eq. (3) do not take into account the quintic nonlinearity, which in the context of short pulse propagation in nonlinear media would correspond to a non-Kerr nonlinearity. By including this term we have obtained a broader class of solutions of Eq. (1) called wide localized (in space) solitons that lends its name to the title of this article. Although we have focused our attention on the propagation of ultrashort pulse propagation described by nonautonomous NLSE, we have found that example 2 may as well be applied to the case of BECs with injection and drain of particles in different sides of a doublewell trapping potential, as described originally in Ref. [27].
The method is general enough and can be applied to obtain exact solutions of other potentials if the solution associated with the NLSE with constant cubic and quintic nonlinearities is known. We would like to mention that whether the original CQNLSE Eq. (1) will be $\mathcal{P} \mathcal{T}$ symmetric or not depends on several factors, crucial among them being the choice of the function $\gamma(Z)$. Here we have chosen a particular form of $\gamma(Z)$, although there are many other choices which would ensure $\mathcal{P} \mathcal{T}$ symmetry, that is, symmetry under $X$ and $Z$ reversals of Eq. (1). Remarkably, we have noticed that such a symmetry, together with the lack of autonomy of (1), is important to guarantee the propagation, without amplification or attenuation of stable solitons.

One could ask about the possibility of experimentally observed systems like those reported in this work, for instance, whether it is necessary to implement the coefficients appearing in the differential equation with a high accuracy . Regarding the nonlinear parameters, it can be said that one can perform an arbitrary scaling in the amplitude of the solution, such that the value of the parameter can be continuously changed for any nonvanishing value. However, the relation between those parameters shall be kept in order to maintain the solutions analytical. The same can be said about the other parameters appearing in the linear terms of the differential equation, but now the scaling shall be done in the coordinate variables. Thus, in a real experimental situation, one could adjust some of the parameters and, even if it is not possible to do this with all of them, the exact analytical solution could be used in order to implement an approximation procedure. Furthermore, since we have verified the stability of some of the solutions found here, one could expect that even in the case that the parameters are not strictly equal to those of the exact solutions, the solitons still will be present in the system.

\section{ACKNOWLEDGMENTS}

L.E.A.M. would like to thank to Dr. L. B. Castro for drawing attention to Ref. [38] and the CAPES/CNPq-IEL NacionalBrasil program for support through a scholarship. P.R. wishes to thank UNESP for supporting a visit to UNESP-Campus de Guaratinguetá during which this work started. This work is also partially supported by CNPq (Grants No. 482043/2011-3, No. 304252/2011-5, and No. 306316/2012-9).
[1] F.-J. Niedernostheide, Nonlinear Dynamics and Pattern Formation in Semiconductors and Devices (Springer, Berlin, 1995).

[2] M. F. S. Ferreira, Nonlinear Effects in Optical Fibers (Wiley \& Sons, Hoboken, NJ, 2011).

[3] F. Dalfovo, S. Giorgini, L. P. Pitaevskii, and S. Stringari, Rev. Mod. Phys. 71, 463 (1999).

[4] M. Kono and M. M. Škorić, Nonlinear Physics of Plasma (Springer, Berlin, 2010).

[5] N. Akhmediev and A. Ankiewicz (eds.), Dissipative Solitons (Springer, Heidelberg, 2008).
[6] V. I. Kruglov, A. C. Peacock, and J. D. Harvey, Phys. Rev. Lett. 90, 113902 (2003); V. N. Serkin, A. Hasegawa, and T. L. Belyaeva, ibid. 98, 074102 (2007); N. Serkin, A. Hasegawa, and T. L. Belyaeva, J. Mod. Opt. 57, 1456 (2010).

[7] N. Akhmediev, M. P. Das, and A. Vagov, Int. J. Mod. Phys. B 13, 625 (1999).

[8] D. A. Zezyulin, G. L. Alfimov, and V. V. Konotop, Phys. Rev. A 81, 013606 (2010); D. A. Zezyulin and V. V. Konotop, ibid. 85, 043840 (2012).

[9] R. Radhakrishnan and M. Lakshmanan, Phys. Rev. E 54, 2949 (1996) 
[10] V. N. Serkin and A. Hasegawa, Phys. Rev. Lett. 85, 4502 (2000); V. N. Serkin, T. L. Belyaeva, I. V. Alexandrov, and G. M. Melchor, Proc. SPIE 4271, 292 (2001).

[11] J. Belmonte-Beitia, V. M. Perez-Garcia, V. Vekslerchik, and V. V. Konotop, Phys. Rev. Lett. 100, 164102 (2008).

[12] A. T. Avelar, D. Bazeia, and W. B. Cardoso, Phys. Rev. E 79, 025602(R) (2009).

[13] L. E. Arroyo Meza, A. de Souza Dutra, and M. B. Hott, Phys. Rev. E 86, 026605 (2012); 88, 053202 (2013).

[14] C. M. Bender and S. Boettcher, Phys. Rev. Lett. 80, 5243 (1998).

[15] Z. Ahmed, Phys. Lett. A 282, 343 (2001).

[16] C. M. Bender, D. C. Brody, and H. F. Jones, Phys. Rev. Lett. 89, 270401 (2002); Am. J. Phys. 71, 1095 (2003).

[17] C. M. Bender, D. C. Brody, H. F. Jones, and B. K. Meister, Phys. Rev. Lett. 98, 040403 (2007).

[18] R. El-Ganainy, K. G. Markis, D. N. Christodoulides, and Z. H. Musslimani, Opt. Lett. 32, 2632 (2007); K. G. Makris, R. El-Ganainy, D. N. Christodoulides, and Z. H. Musslimani, Phys. Rev. Lett. 100, 103904 (2008); A. Guo, G. J. Salamo, D. Duchesne, R. Morandotti, M. Volatier-Ravat, V. Aimez, G. A. Siviloglou, and D. N. Christodoulides, ibid. 103, 093902 (2009); C. E. Rüter, K. G. Makris, R. El-Ganainy, D. N. Christodoulides, M. Segev, and D. Kip, Nat. Phys. 6, 192 (2010); T. Kottos, ibid. 6, 166 (2010); H. Ramezani, T. Kottos, R. El-Ganainy, and D. N. Christodoulides, Phys. Rev. A 82, 043803 (2010); O. Bendix, R. Fleischmann, T. Kottos, and B. Shapiro, J. Phys. A 43, 265305 (2010).

[19] E. M. Graefe, H. J. Korsch, and A. E. Niederle, Phys. Rev. Lett. 101, 150408 (2008).

[20] H. Cartarius and G. Wunner, Phys. Rev. A 86, 013612 (2012).

[21] A. Sinha and P. Roy, Int. J. Mod. Phys. A 21, 5807 (2006); V. G. C. S. dos Santos, A. de Souza Dutra, and M. B. Hott, Phys. Lett. A 373, 3401 (2009); S. Longhi, Phys. Rev. Lett. 105, 013903 (2010); L. B. Castro, Phys. Lett. A 375, 2510 (2011).

[22] C. Dembowski, B. Dietz, H. D. Graf, H. L. Harney, A. Heine, W. D. Heiss, and A. Richter, Phys. Rev. Lett. 90, 034101 (2003).

[23] A. E. Siegman, Laser (University Science Books, Sausalito, CA, 1986); E. A. Ultanir, G. I. Stegeman, and D. N. Christodoulides, Opt. Lett. 29, 845 (2004).

[24] Z. H. Musslimani, K. G. Makris, R. El-Ganainy, and D. N. Christodoulides, Phys. Rev. Lett. 100, 030402 (2008).

[25] Z. H. Musslimani, K. G. Makris, R. El-Ganainy, and D. N. Christodoulides, J. Phys. A 41, 244019 (2008); K. G. Makris, R. El-Ganainy, D. N. Christodoulides, and Z. H. Musslimani, Int. J. Theor. Phys. 50, 1019 (2008).
[26] S. Hu, X. Ma, D. Lu, Z. Yang, Y. Zheng, and W. Hu, Phys. Rev. A 84, 043818 (2011); C. Moreira, F. Kh. Abdullaev, V. V. Konotop, and A. V. Yulin, ibid. 86, 053815 (2012); S. Hu and W. Hu, J. Phys. B 45, 225401 (2012); Eur. Phys. J. D 66, 266 (2012); Z. Chen, M. Segev, and D. N. Christodoulides, Rep. Prog. Phys. 75, 086401 (2012); I. V. Barashenkov, S. V. Suchkov, A. A. Sukhorukov, S. V. Dmitriev, and Y. S. Kivshar, Phys. Rev. A 86, 053809 (2012); H. Cartarius, D. Haag, D. Dast, and G. Wunner, J. Phys. A 45, 444008 (2012); Y. Meng and Y. Liu, J. Opt. Soc. Am. B 30, 1148 (2013).

[27] S. Klaiman, U. Günther, and N. Moiseyev, Phys. Rev. Lett. 101, 080402 (2008).

[28] S. A. Ponomarenko and G. P. Agrawal, Phys. Rev. Lett. 97, 013901 (2006); Opt. Lett. 32, 1659 (2007); C. Dai, Y. Wang, and C. Yan, Opt. Commun. 283, 1489 (2010); T. S. Raju and P. K. Panigrahi, Phys. Rev. A 81, 043820 (2010); Z. -Y. Yang, L.-C. Zhao, T. Zang, Y.-H. Li, and R.-H. Yue, Opt. Commun. 283, 3768 (2010).

[29] Z. Shi, X. Jiang, X. Zhu, and H. Li, Phys. Rev. A 84, 053855 (2011).

[30] A. de Souza Dutra, M. B. Hott, and V. G. C. S. dos Santos, Europhys. Lett. 71, 166 (2005).

[31] Jun-Rong He and Hua-Mei Li, Phys. Rev. E 83, 066607 (2011).

[32] C. Farina de Souza and A. de Souza Dutra, Phys. Lett. A 123, 297 (1987).

[33] E. Merzbacher, Quantum Mechanics (Wiley \& Sons, New York, 1998); B. Chern and A. Tubis, Am. J. Phys. 35, 254 (1967).

[34] M. Abramowitz and I. Setgun, Hanbook of Mathematical Functions (Dover, Toronto, 1965).

[35] Y. V. Kartashov, L. Torner, and V. A. Vysloukh, Opt. Lett. 29, 1102 (2004); R. Yang and X. Wu, Opt. Express 16, 17759 (2008).

[36] B. Midya and R. Roychoudhury, Phys. Rev. A 87, 045803 (2013).

[37] M. Znojil, J. Phys. A 33, L61 (2000); G. Lévai and E. Magyari, ibid. 42, 195302 (2009).

[38] A. Khare, S. M. Al-Marzoug, and H. Bahlouli, Phys. Lett. A 376, 2880 (2012).

[39] W. B. Cardoso, A. T. Avelar, and D. Bazeia, Phys. Rev. E 86, 027601 (2012)

[40] Q. Chang, E. Jia, and W. Sun, J. Comp. Phys. 148, 397 (1999).

[41] D. Dubin, Numerical and Analytical Methods for Scientists and Engineers Using Mathematica (Wiley \& Sons, Hoboken, NJ, 2003).

[42] A. M. Lyapunov, General Problem of the Stability Of Motion (Taylor \& Francis, London, 1992).

[43] L. Elsgoltz, Ecuaciones Diferenciales y Cálculo Variacional (Editorial Mir, Moscow, 1969). 